\title{
Double beta decay and neutrino mass models
}

\author{
J.C. Helo, ${ }^{a}$ M. Hirsch, ${ }^{b}$ T. Ota ${ }^{c}$ and F.A. Pereira dos Santos ${ }^{d}$ \\ ${ }^{a}$ Universidad Técnica Federico Santa María, \\ Centro-Científico-Tecnológico de Valparaíso, \\ Casilla 110-V, Valparaíso, Chile \\ ${ }^{b}$ AHEP Group, Instituto de Física Corpuscular - C.S.I.C./Universitat de València, \\ Edificio de Institutos de Paterna, Apartado 22085, E-46071 València, Spain \\ ${ }^{c}$ Department of Physics, Saitama University, \\ Shimo-Okubo 255, 338-8570 Saitama-Sakura, Japan \\ ${ }^{d}$ Departamento de Física, Pontifícia Universidade Católica do Rio de Janeiro, \\ Rua Marquês de São Vicente 225, 22451-900 Gávea, Rio de Janeiro, Brazil \\ E-mail: juancarlos.helo@usm.cl, mahirsch@ific.uv.es, \\ toshi@mail.saitama-u.ac.jp, fabio.alex@fis.puc-rio.br
}

Abstract: Neutrinoless double beta decay allows to constrain lepton number violating extensions of the standard model. If neutrinos are Majorana particles, the mass mechanism will always contribute to the decay rate, however, it is not a priori guaranteed to be the dominant contribution in all models. Here, we discuss whether the mass mechanism dominates or not from the theory point of view. We classify all possible (scalar-mediated) short-range contributions to the decay rate according to the loop level, at which the corresponding models will generate Majorana neutrino masses, and discuss the expected relative size of the different contributions to the decay rate in each class. Our discussion is general for models based on the SM group but does not cover models with an extended gauge. We also work out the phenomenology of one concrete 2-loop model in which both, mass mechanism and short-range diagram, might lead to competitive contributions, in some detail.

Keywords: Beyond Standard Model, Neutrino Physics

ARXIV EPRINT: 1502.05188 


\section{Contents}

1 Introduction 1

2 Setup and classification $\quad 5$

2.1 Generalities 5

$\begin{array}{lll}2.2 & \text { Tree-level neutrino mass models } & 7\end{array}$

2.3 1-loop models 9

2.4 2-loop models 11

$\begin{array}{lll}2.5 & 3 \text {-loop models } & 14\end{array}$

$\begin{array}{lll}2.6 & \text { 4-loop models } & 17\end{array}$

3 A concrete 2-loop example 18

3.1 General formulas for neutrino masses and $\mu \rightarrow e \gamma \quad 19$

3.2 One generation of $\psi_{6,2,1 / 6} \quad 21$

3.3 Three generations of $\psi_{6,2,1 / 6} \quad 24$

4 Conclusions and discussion $\quad 26$

$\begin{array}{lr}\text { A Summary tables } & 28\end{array}$

\section{Introduction}

Experimental limits on half-lives of neutrinoless double beta decay $(0 \nu \beta \beta)$ give stringent bounds on many Lepton Number Violating (LNV) extensions of the Standard Model (SM); for a recent review see, for example [1]. Recent experimental results give limits for ${ }^{76} \mathrm{Ge}[2]$ and ${ }^{136} \mathrm{Xe}[3-5]$ in excess of $10^{25} \mathrm{ys}$, which place an upper limit on the effective Majorana mass $\left\langle m_{\nu}\right\rangle^{1}$ of the order of roughly $\left\langle m_{\nu}\right\rangle \lesssim(0.2-0.4) \mathrm{eV}$, depending on calculations of nuclear matrix element [6-8].

However, from the theoretical point of view it is not a priori clear, whether the mass mechanism gives indeed the dominant contribution to the double beta decay rate, and many models have been discussed in the literature where this might not be the case. The classical example appears in left-right (LR) symmetric extensions of the SM $[9,10]$ and also in R-parity violating $\left(\not R_{P}\right)$ supersymmetric theories with both trilinear $\not R_{P}[11,12]$ and bilinear $\not R_{P}[13,14]$ terms. Furthermore, leptoquark models [15] and more recently models with colour octet scalars [16] or colour sextet diquarks [17-19] have been discussed.

\footnotetext{
${ }^{1}\left\langle m_{\nu}\right\rangle$ is defined as $\left\langle m_{\nu}\right\rangle=\sum_{j} U_{e j}^{2} m_{j}$, where the sum runs over all light neutrinos. This is equivalent to the $(e, e)$ entry of the Majorana neutrino mass matrix in the basis where the charged lepton mass matrix is diagonal.
} 
Given that there is such a large list of possible lepton number violating models, is it possible to determine which contribution to the $0 \nu \beta \beta$ decay rate is the dominant one? - Perhaps, if double beta decay were to be observed in the next round of experiments and either KATRIN [20] or cosmological data [21-23] also find hints for a neutrino mass scale of the order of, say, somewhat larger than $\mathcal{O}(0.1) \mathrm{eV}$, one could claim on the basis of minimality that the mass mechanism $\left\langle m_{\nu}\right\rangle$ gives (at least) the most important contribution to the total decay rate. However, once upper limits on the total neutrino mass $\left(\sum m_{\nu}\right)$ placed from cosmology drop below the level of $\mathcal{O}(0.1) \mathrm{eV}$, the question becomes exceedingly difficult to answer.

In that case, from the experimental point of view, there remain only a few possibilities to make progress. For example, measurements of the angular correlation between the two electrons from $0 \nu \beta \beta[10,24,25]$ or measuring double beta plus decays [26] offer the possibility to identify the Lorentz structure (equivalently the chiralities of the emitted electrons) of the LNV processes. However, realistically the SuperNEMO proposal [25] could only accumulate the necessary statistics to identify the Lorentz structure, if the half-live of ${ }^{82} \mathrm{Se}$ is below $10^{26}$ ys, while there yet exists no experimental proposal with a sufficient sensitivity for $0 \nu \beta^{+} / E C$ decays to make use of the ideas discussed in [26].

From the theoretical point of view, as discussed in [27, 28], the amplitude of $0 \nu \beta \beta$ decay can be divided into a long-range and a short-range part. Here, short-range means that all particles appearing in the diagrams for double-beta decay are heavier than the nuclear Fermi scale, i.e. $\mathcal{O}(0.1) \mathrm{GeV}$. Current limits from $0 \nu \beta \beta$ decay then correspond to lower limits on the effective scale $\Lambda_{\mathrm{LNV}}$ of lepton number violation,

$$
\Lambda_{\mathrm{LNV}} \equiv\left(\frac{m_{S}^{4} m_{F}}{g_{\mathrm{eff}}^{4}}\right)^{1 / 5} \gtrsim(1-3) \mathrm{TeV},
$$

where $g_{\text {eff }}$ is some mean of the couplings appearing in the diagram and $m_{S}$ and $m_{F}$ are the masses of the fields that mediate the $0 \nu \beta \beta$ process, see the next section for details. This mass scale is testable, at least in principle, at the LHC, and the combination of future LHC limits (or a possible discovery, to express it in a more optimistic way) and double beta decay data might allow to test many, but maybe not all, of the possible short-range diagrams that contribute to the decay rate $[29,30]$.

In this paper we take a different approach and study the question, whether the mass mechanism is dominant or not, from a purely theoretical point of view. As described above, current and next generation $0 \nu \beta \beta$ decay experiments will test LNV interactions at the TeV scale. Such TeV-scale LNV interactions, on the other hand, appear also in the context of radiative neutrino mass models. In other words, a new physics (short-range) contribution to $0 \nu \beta \beta$ decay will always also produce a non-zero neutrino mass. In this paper we discuss the relation between possible models for short-range contribution to $0 \nu \beta \beta$ decay and the neutrino mass-generation mechanism. Our study is based on the complete list of possible decompositions of the $d=9$ (short-range) double beta decay operator given in [31]. The general decomposition list given in [31] is equivalent, in principle, to defining all models which can give a contribution to double beta decay, and the black box theorem, see below, guarantees that all these models will produce Majorana neutrino masses at or below four- 


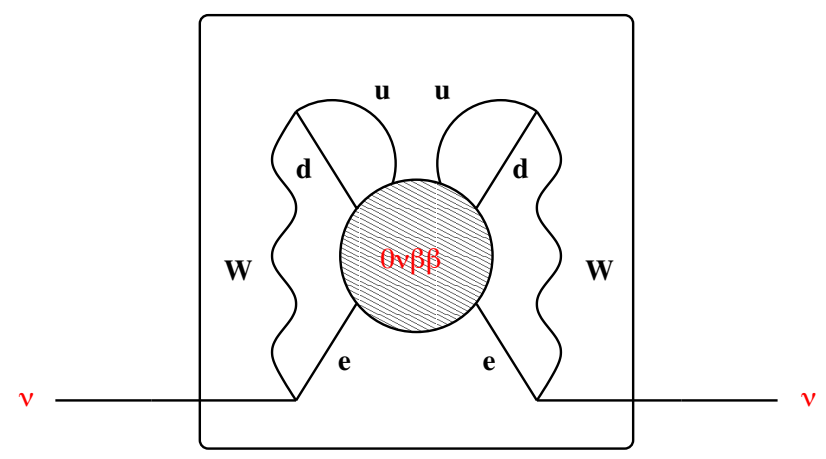

Figure 1. Schematic explanation of the black box theorem: the theorem guarantees that, once $0 \nu \beta \beta$ decay has been observed, Majorana neutrino masses will be generated, independent of the underlying model, at the latest at the four-loop level. By itself, this theorem does not guarantee that the mass mechanism is the dominant contribution to $0 \nu \beta \beta$ decay.

loop order. Our approach therefore basically consists in classifying all the possible models contributing to $0 \nu \beta \beta$ decay with respect to the loop level at which they will generate Majorana neutrino masses. We can then discuss the expected size of the two contributions to $0 \nu \beta \beta$ : (1) the $d=9$ short-range contribution $\mathcal{O}_{d=9}$ and (2) the contribution from the neutrino mass mechanism $\left\langle m_{\nu}\right\rangle$ which is radiatively induced in the corresponding models and conclude model-by-model, which one of the two is expected to dominate. Given that the list of [31] is tree-level complete, our discussion is quite general and covers actually models of neutrino mass from tree-level models to 4-loop models.

Before entering into the details of our work, let us briefly comment on the well-known relation between short-range $0 \nu \beta \beta$ contributions and neutrino masses, i.e., the black box theorem [32]. ${ }^{2}$ The theorem guarantees that, once $0 \nu \beta \beta$ decay has been observed, neutrinos are Majorana particles, see figure 1. However, the black box theorem does not demonstrate that the mass mechanism dominates $0 \nu \beta \beta$, since it only guarantees neutrino masses at the level of four-loop. Obviously, a four-loop diagram, additionally suppressed by $m_{u}^{2} m_{d}^{2} m_{e}^{2} / \Lambda_{\mathrm{LNV}}^{5}$, can produce only tiny neutrino masses, which are many orders of magnitude below of what is required to explain oscillation data [34]. Nevertheless, the black box theorem, together with the general decomposition of the $d=9$ double beta decay operator published in [31], defines the basic idea of our current paper. Indeed we find that all "models" listed in [31] produce neutrino masses at or below the 4-loop order as demanded by the theorem.

We comment that our work has some overlap with [35, 36] and [37]. Babu \& Leung [35] have written down all SM invariant $\Delta L=2$ operators from dimension- 5 ( $d=5$, the wellknown Weinberg operator [38]) to $d=11$ and showed the relation between the effective operators and neutrino masses on the basis of black-box like loop diagrams. The authors of $[35,36]$ discuss then possible ultra-violet completions for several example operators and give estimates for the scales $\Lambda_{\mathrm{LNV}}$, for which those operators can explain current neutrino

\footnotetext{
${ }^{2}$ In [33] an extension of the black box mechanism with flavour violation has been constructed.
} 
data. The authors of [37] provide a systematic study of these operators, for one-loop and two-loop neutrino mass models, and discuss also which of these could possibly be tested at the LHC. However, our discussion differs from these papers in that we are mostly interested in double beta decay and its relation to neutrino mass.

We mention also the work of [39], which pursues the link between the short-range contribution to $0 \nu \beta \beta$ and neutrino masses, but takes a different approach from ours. The authors focus on three types of LNV effective interactions which consist only of leptons and Higgs doublets, and list the models in which those LNV interactions simultaneously drive both the new physics contribution to $0 \nu \beta \beta$ and neutrino masses. The main difference between [39] and our work is that it is assumed in [39] that new physics resides in the leptonic sector only.

In our classification, we also rediscover several models discussed in the literature previously, like for example leptoquark models [15], which can give 1-loop neutrino masses as discussed in [40] or 2-loop neutrino masses, as in the model of [19] or the one in [41]. We do not cover, however, the possible contributions from light sterile neutrinos. There exists a vast amount of papers on this subject in the literature already [42-54] and we have nothing new to add on this particular subject.

Since neutrino mass models must not only produce the correct absolute values of neutrino mass, but also reproduce the observed flavour structure of the neutrino mass matrix, one also has to pay attention to constraints from flavour physics observables. In [55] the authors applied the hypothesis of "minimal flavour violation" (MFV) to effective operators that contribute to $0 \nu \beta \beta$ and found that the MFV assumption constrains the effective operators to be smaller than the detectable level. In this work, we do not adopt any such theoretical assumption on the flavour structures of the parameters in the models. Instead we simply consider bounds on lepton flavour violating observables as constraints on model parameters. We believe this to be the correct approach since any of the "exotic" contributions to $0 \nu \beta \beta$ decay requires the introduction of new scalars, not present in the SM, with their own Yukawa interactions with SM fermions. Thus the whole concept of MFV is not very well funded in any of the models of interest for $0 \nu \beta \beta$ decay.

A few disclaimers might also be in order here. Our analysis concentrates on the true $d=9$ operator, i.e. it covers only the short range part of the $0 \nu \beta \beta$ amplitude. Our results thus do not cover, for example, the long-range diagrams of R-parity violating SUSY [56, 57] or leptoquark models [15]. Also, we limit ourselves to scalar exchange, thus models with a coupling between new scalars and the SM gauge bosons, such as [58, 59] are not considered. Also, this restrictions implies that we do not cover models with an extended gauge group either, especially we do not discuss models with left-right symmetry. And, finally, the list of decompositions in [31] is complete only at tree-level. Thus, we do not consider cases in which the neutrino mass is generated at some higher loop level, while the $0 \nu \beta \beta$ amplitude appears at one-loop order, as for example in the recent papers [60, 61].

The rest of this paper is organised as follows. At the beginning of section 2 , as a preparation, we will summarise the main results of [31] and discuss some generalities useful for the latter parts of the paper. We will then discuss the classification of the different possible models and estimate in each case the relative size of the contribution from the 
mass mechanism and the short range part of the amplitude. In section 3, we will discuss one concrete two-loop model of neutrino mass in more detail. Section 4 summarises and discusses our main findings. Tables with lists of the different models, classified as described in section 2 are deferred to the appendix.

\section{Setup and classification}

In this section, we classify neutrino mass models based on the decomposition of the $d=9$ $0 \nu \beta \beta$ effective operators, according to the number of loops in the resulting neutrino mass diagrams. In each class, we will compare the size of the two contributions to the $0 \nu \beta \beta$ : (i) $d=9$ operator itself, and (ii) the mass mechanism $\left\langle m_{\nu}\right\rangle$ induced by the $d=9$ operator. This classification therefore allows to identify those models, for which one can expect nonstandard contributions (beyond the ordinary mass mechanism) to be important for $0 \nu \beta \beta$ decay. Note, that $\left\langle m_{\nu}\right\rangle$, is equivalent to the e-e entry in the neutrino mass matrix, $\left(M_{\nu}\right)_{e e}$, in the basis where the charged lepton mass matrix is diagonal. Thus, in this section we concentrate on the comparison of short-range contributions to the size of this entry in $\left(M_{\nu}\right)$. Of course, a complete fit to all neutrino data will need to take into account also all other entries in $\left(M_{\nu}\right)$. A specific example, how this can be done and the additional constraints from both, oscillation data and lepton flavour violating decays, is discussed in section 3 . In the discussion in this section, we always keep generation indices in the unknown couplings of the different models. Other indices could be constrained combining double beta decay with, for example, oscillation data. A specific example for this is worked out in section 3.

\subsection{Generalities}

The short-range double beta decay operator

$$
\mathcal{O}_{d=9} \propto \bar{u} \bar{u} d d \bar{e} \bar{e}
$$

can be generated at tree-level via only two topologies shown in figure 2. The bosons (depicted with dashed lines in the diagrams) in these topologies could be either scalars or vectors, but we will consider only scalar exchange here. Assigning the outer fermions with either the left (" $L$ ") or the right (" $R$ ") chirality in all possible permutations allows to derive the complete list of "decompositions" (or proto-models) that can contribute to the $0 \nu \beta \beta$ decay amplitude at tree level [31]. The fermion propagator in topology-I contains two terms,

$$
\frac{\not p+m_{\psi}}{p^{2}-m_{\psi}^{2}}
$$

but in the short-range part of the amplitude the first term is suppressed relative to the second by a factor of $\not p / m_{\psi} \simeq p_{F} / m_{\psi}$, where $p_{F}$ is the typical Fermi momentum in the nucleus and the mass $m_{\psi}$ is suppossed to be larger than $\mathcal{O}(100) \mathrm{GeV}$. Considering then only decompositions which pick the mass term from the propagator results in a total of 135 possible decompositions for Topology-I (T-I in the following), while there are 27 decompositions in Topology-II (T-II), if we limit ourselves to scalar exchange [31]. For tables showing the different decompositions see the appendix. 

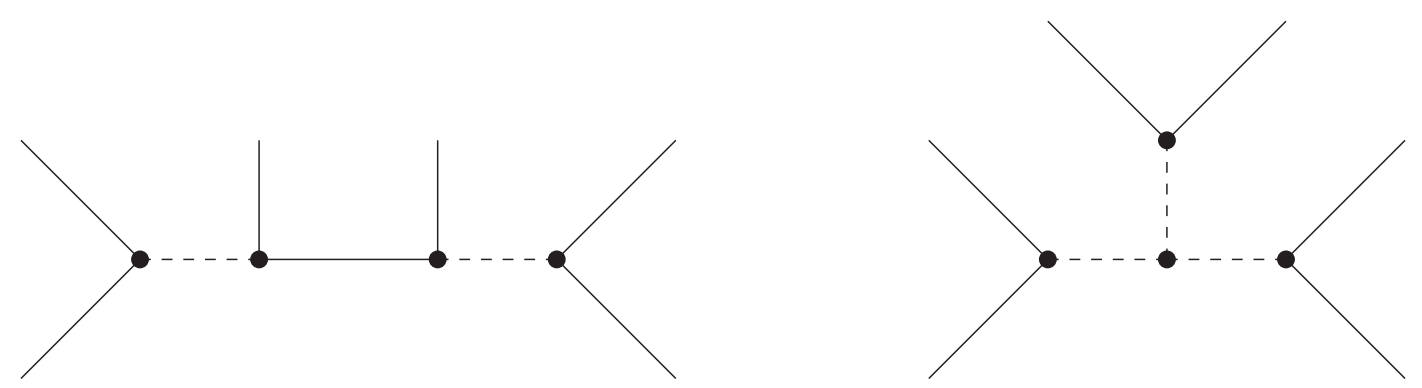

Figure 2. The two possible tree-level topologies contributing to the $0 \nu \beta \beta$ decay rate. The outer lines represent the six SM fermions, while for the virtual particles appearing in the inner lines we consider only scalar-fermion-scalar (left, topology-I) and scalar-scalar-scalar (right, topology-II).

Babu \& Leung [35] have listed $\Delta L=2$ operators from $d=5$ to $d=11$. Among the $d=9$ operators in their list, the following five are relevant for double beta decay:

$$
\begin{array}{lll}
\mathcal{O}_{11}=\bar{L} \bar{L} \bar{Q} d_{R} \bar{Q} d_{R}, & \mathcal{O}_{12}=\bar{L} \bar{L} \overline{u_{R}} Q \overline{u_{R}} Q, & \mathcal{O}_{14}=\bar{L} \bar{L} \overline{u_{R}} Q \bar{Q} d_{R}, \\
\mathcal{O}_{19}=\bar{L} \overline{e_{R}} \bar{Q} d_{R} \overline{u_{R}} d_{R}, & \mathcal{O}_{20}=\bar{L} \overline{e_{R}} \overline{u_{R}} Q \overline{u_{R}} d_{R} . &
\end{array}
$$

Here, we have suppressed the indices of generation, $\mathrm{SU}(3)_{c}, \mathrm{SU}(2)_{L}$, and Lorentz spinor, which are contracted appropriately. In the list of decompositions shown in [31], there appears also one operator not given in [35], which is ${ }^{3}$

$$
\mathcal{O}_{-}=\overline{e_{R}} \overline{e_{R}} \overline{u_{R}} d_{R} \overline{u_{R}} d_{R} .
$$

As the black box theorem demonstrates, one can obtain the Weinberg operator by connecting the quark legs in these effective operators with the SM Yukawa interactions. From the effective operator point of view it seems that neutrino masses are generated at 2-loop level from $\mathcal{O}_{11}, \mathcal{O}_{12}$ and $\mathcal{O}_{14}$. The operators $\mathcal{O}_{19}$ and $\mathcal{O}_{20}$ need an additional SM Yukawa interaction with a charged lepton to generate a neutrino mass term, thus they end up with 3-loop diagrams. The operator $\mathcal{O}_{-}$leads to neutrino masses only at the 4-loop level, which is equivalent to the original black box diagram. However, the classification of the neutrino mass models with respect to the number of loops, which we discussed in the introduction, is modified from this naive expectation, once the decomposition of the operators are specified. In fact, as shown below, many decompositions of the operators in eq. (2.3) contain automatically the particle content (and interactions) such that neutrino masses are generated at lower order, i.e., both tree-level and 1-loop neutrino mass models are found. And, surprisingly, also the opposite case exists: if we restrict ourselves to decomposing the operators only with scalar and fermion mediators, none of the decompositions of the operator $\mathcal{O}_{14}$ generates a genuine neutrino mass diagram at the 2-loop level. We will come back to this important point later in more detail. Genuineness is one of the key concepts in our classification method. The term, genuine n-loop neutrino mass model, is defined as

\footnotetext{
${ }^{3}$ As discussed below, this operator induces neutrino mass only at the four-loop level. Probably for that reason it was neglected in [35].
} 

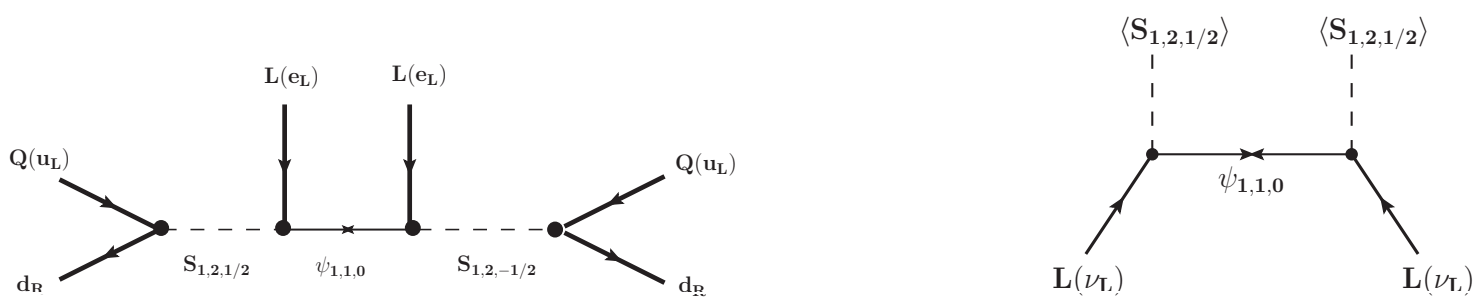

Figure 3. To the left: diagram for $0 \nu \beta \beta$ decay via charged scalar exchange for Babu-Leung operator $\mathcal{O}_{11}$ (BL\#11). To the right: tree-level neutrino mass generated via seesaw type-I, using the same vertices as in the diagram on the left. Here and in all Feynman diagrams below, arrows on fermion lines indicate the flow of particle number, not the chirality of the fermion.

the model in which the neutrino mass term is generated at the $n$-loop level and for which, simultaneously, diagrams with loop level lower than $n$ are guaranteed to be absent, see [62] for more details.

\section{$2.2 \quad$ Tree-level neutrino mass models}

Let us start with a rather trivial example, illustrated in figure 3 , in which the $d=9$ contribution to $0 \nu \beta \beta$ is related to the Majorana neutrino mass at the tree level. Here, as everywhere else in this paper, subscripts on fields denote their transformation properties (or charge in case of $\left.\mathrm{U}(1)_{Y}\right)$ under the $\mathrm{SM}$ group, $\mathrm{SU}(3)_{c} \times \mathrm{SU}(2)_{L} \times \mathrm{U}(1)_{Y}$. A new scalar field is denoted by the symbol $S$, and a fermion field, which is understood as either a vector-like fermion or Majorana fermion, is denoted as $\psi$. Thus, $\psi_{1,1,0}$ has the same quantum numbers as a right-handed neutrino, while $S_{1,2,1 / 2}$ is equivalent to a (copy of) the SM Higgs doublet.

The Lagrangian producing the left diagram of figure 3 necessarily contains the following terms:

$$
\mathcal{L}=\left(Y_{S Q d}\right)_{i j} \bar{Q}_{i} \cdot S_{1,2,1 / 2} d_{R, j}+\left(Y_{S \nu \psi}\right)_{i} \overline{L_{i}} \cdot S_{1,2,1 / 2}^{\dagger} \psi_{1,1,0}+m_{\psi} \overline{\left(\psi_{1,1,0}\right)^{c}} \psi_{1,1,0}+\text { H.c. }
$$

Here, the singlet fermion field $\psi_{1,1,0}$ is allowed to have a Majorana mass $m_{\psi}$. The dot (.) denotes the anti-symmetric tensor $\left(\mathrm{i} \tau^{2}\right)$ for $\mathrm{SU}(2)_{L}$. This Lagrangian generates an effective operator for a short-range contribution to $0 \nu \beta \beta$,

$$
\mathcal{L}_{d=9}=\frac{\left(\left(Y_{S Q d}\right)_{11}\left(Y_{S \nu \psi}\right)_{e}\right)^{2}}{m_{S_{1,2,1 / 2}}^{4} m_{\psi}}\left(\bar{Q} d_{R}\right) \cdot \bar{L} L^{c} \cdot\left(\bar{Q} d_{R}\right)+\text { H.c. },
$$

which corresponds to $\mathcal{O}_{1}^{S R}$ in the notation of [28]. (Here we use the notation $\mathcal{O}_{i}^{S R}$ for the five relevant short-range operators, defined in [28], to distinguish them from the lepton number violating operators $\mathcal{O}_{j}, j=11,12,14,19,20$ and "-".) $m_{S_{1,2,1 / 2}}$ is the mass of $S_{1,2,1 / 2}$. Following the method adopted in $[1,31]$ and using the experimental bound [3-5]

$$
T_{1 / 2}^{0 \nu \beta \beta}\left({ }^{136} \mathrm{Xe}\right)>1.6 \cdot 10^{25}[\mathrm{ys}]
$$

one finds the bound on the coefficient of the $d=9$ operator as

$$
\frac{1}{8} \frac{\left(\left(Y_{S Q d}\right)_{11}\left(Y_{S \nu \psi}\right)_{e}\right)^{2}}{m_{S_{1,2,1 / 2}}^{4} m_{\psi}}=\frac{G_{F}^{2}}{2 m_{P}} \epsilon_{1}^{\{R R\} R} \lesssim \frac{G_{F}^{2}}{2 m_{P}} 2.6 \cdot 10^{-7},
$$


which can be interpreted as a constraint on the Yukawa coupling:

$$
\left(Y_{S \nu \psi}^{2}\right)_{e} \lesssim 1.5 \cdot 10^{-6}\left(\frac{1.0}{Y_{S Q d}^{2}}\right)\left(\frac{m_{S_{1,2,1 / 2}}}{100[\mathrm{GeV}]}\right)^{4}\left(\frac{m_{\psi}}{100[\mathrm{GeV}]}\right) .
$$

If the scalar mediator $S_{1,2,1 / 2}$ acquires a vacuum expectation value (vev), the right diagram in figure 3 , which has the same topology as the type-I seesaw mechanism, will contribute to neutrino mass(es):

$$
\left\langle m_{\nu}\right\rangle=\frac{\left(Y_{S \nu \psi}^{2}\right)_{e}\langle S\rangle^{2}}{m_{\psi}}
$$

The experimental bound on the effective neutrino mass $\left\langle m_{\nu}\right\rangle \lesssim 0.3 \mathrm{eV}$, which is found from eq. (2.7) under the assumption of the mass mechanism being dominant, gives

$$
\left(Y_{S \nu \psi}^{2}\right)_{e} \lesssim 1.0 \cdot 10^{-12}\left(\frac{m_{\psi}}{100[\mathrm{GeV}]}\right)\left(\frac{v_{\mathrm{SM}}}{\langle S\rangle}\right)^{2}\left(\frac{\left\langle m_{\nu}\right\rangle}{0.3[\mathrm{eV}]}\right)
$$

where we have used $v_{\mathrm{SM}} \simeq 174 \mathrm{GeV}$. If $S_{1,2,1 / 2}$ is identified as the SM Higgs doublet $H$, as in the ordinary type-I seesaw, the constraint on $Y_{S \nu \psi}$ shown in eq. (2.11) is obviously much stronger than eq. (2.9). ${ }^{4}$ In other words: the mass mechanism dominates the contribution to $0 \nu \beta \beta$ decay by far, if we consider SM Higgs exchange.

However, $S_{1,2,1 / 2}$ is not necessarily the SM Higgs, it could be an additional new state, such as appear, for example, in multi-Higgs doublet models. In this case, neutrino masses would still be generated through the type-I seesaw mechanism, with the vev $\langle S\rangle$ of the scalar $S_{1,2,1 / 2}$ independent of the SM vev $v_{\mathrm{SM}}$, such as occurs, for example, in the neutrinophilic neutrino mass model of [63-66]. For this case one finds that if the relaxed constraint

$$
\langle S\rangle \lesssim 0.14[\mathrm{GeV}]\left(\frac{\left(Y_{S Q d}\right)_{11}}{1.0}\right)^{2}\left(\frac{100[\mathrm{GeV}]}{m_{S}}\right)^{2}
$$

holds, eq. (2.9) becomes more stringent than eq. (2.11), i.e., the short-range diagram will be the dominant contribution to the $0 \nu \beta \beta$ decay amplitude in this case.

If $S_{1,2,1 / 2}$ has exactly zero vev, in the literature often called the "inert doublet", we can no longer directly relate the the relative size of the $d=9$ operator with the mass mechanism. The only conclusion one can derive in this particular case is the trivial constraint that the standard model Higgs coupling with $L$ and $\psi_{1,1,0}$ must obey eq. (2.11).

As is well-known, there are only three types of tree-level mass generation mechanisms (seesaw mechanisms) called type-I [67-70], type-II [9, 71-75] and type-III [76]. These are mediated by the singlet Majorana fermion $\psi_{1,1,0}$ (type-I), the triplet scalar $S_{1,3,1}$ (type-II), and the triplet Majorana fermion $\psi_{1,3,0}$ (type-III). From the complete list of decompositions given in [31], one can find that T-I-1-i, 2-i-b, 2-ii-b, 2-iii-a, 4-i, and 5-i contain the relevant fermion mediators, and T-I-1-ii-a, 1-ii-b, 3-ii, 3-iii, T-II-1, and T-II-3 do contain the scalar mediator. From the discussion above, we can conclude that for all of these the short-range contribution will be much less important than the neutrino mass mechanism, unless the vev of the new scalars $S_{1,2,1 / 2}$ or $S_{1,3,1}$ are heavily suppressed compared to $v_{\mathrm{SM}}$.

\footnotetext{
${ }^{4}$ If $S_{1,2,1 / 2}$ is the SM Higgs doublet, the coupling $Y_{S Q d}$ is identified as the down quark Yukawa coupling, $Y_{d} \sim 3 \times 10^{-5}$, and the constraint shown in eq. (2.9) actually becomes less stringent than even the ordinary perturbativity bound.
} 

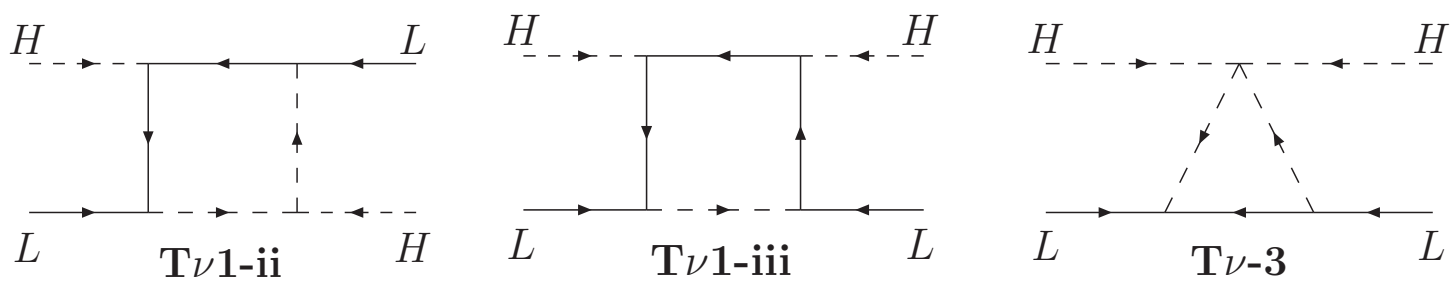

Figure 4. Three different 1-loop diagrams for neutrino mass [77], which can appear in one of the $0 \nu \beta \beta$ decay decompositions. For a discussion see text.

\subsection{1-loop models}

As shown in [77] (see also [78]), there exist a total of four genuine 1-loop diagrams, which can contribute to the $d=5$ Weinberg operator at the renormalizable level. ${ }^{5}$ Three diagrams, shown in figure 4 , can be related to $0 \nu \beta \beta$ decay decompositions. ${ }^{6}$ Here, we have added a " $\nu$ " to the naming conventions of [77], in order not to confuse the 1-loop neutrino mass diagrams with the double beta decay topologies.

Let us discuss the relation between the neutrino mass diagram $\mathrm{T} \nu$-1-ii, which is shown as the left-most diagram in figure 4 , and the decomposition of the $d=90 \nu \beta \beta$ diagram $\left(\overline{u_{L}} d_{R}\right)\left(\overline{e_{L}} d_{R}\right)\left(\overline{u_{L}} \overline{e_{L}}\right)$, which is classified with the ID number T-II-2 $\mathcal{O}_{11}$ in [31], as an example of this class of models. The decomposition leads to a Lagrangian, which contains the following terms:

$$
\begin{aligned}
\mathcal{L}= & \left(Y_{L d S}\right)_{i j} \bar{L}_{i} \cdot\left(S_{3,2,1 / 6}\right)^{\dagger} d_{R, j}+\left(Y_{L Q S}\right)_{i j} \bar{Q}_{j} \cdot L_{i}^{c} S_{3,1,-1 / 3} \\
& +\left(Y_{Q d S}\right)_{i j} \bar{Q}_{i} S_{1,2,1 / 2} d_{R, j}+\mu_{S S S}\left(S_{3,1,-1 / 3}\right)^{\dagger} S_{3,2,1 / 6} S_{1,2,1 / 2}^{\dagger}+\text { H.c. } \\
& +m_{S_{3,2,1 / 6}}^{2}\left(S_{3,2,1 / 6}\right)^{\dagger} S_{3,2,1 / 6}+m_{S_{3,1,-1 / 3}}^{2}\left(S_{3,1,-1 / 3}\right)^{\dagger} S_{3,1,-1 / 3} \cdot+m_{S_{1,2,1 / 2}}^{2}\left(S_{1,2,1 / 2}\right)^{\dagger} S_{1,2,1 / 2} .
\end{aligned}
$$

The lepton number violation can then be assigned to be due to the presence of the coupling $\mu_{S S S}$. With this Lagrangian, eq. (2.13), the effective $d=9$ Lagrangian that contributes to $0 \nu \beta \beta$ process as short-range effects is given as:

$$
\begin{aligned}
\mathcal{L}_{d=9} & =\frac{\left(Y_{L d S}\right)_{e 1}\left(Y_{Q d S}\right)_{11}\left(Y_{L Q S}\right)_{1 e} \mu_{S S S}}{m_{S_{3,2,1 / 6}}^{2} m_{S_{3,1,-1 / 3}}^{2} m_{S_{1,2,1 / 2}}^{2}}\left(\bar{L} d_{R}\right) \cdot\left(\bar{Q} d_{R}\right)\left(\bar{Q} \cdot L^{c}\right)+\text { H.c. } \\
& \supset-\frac{\left(Y_{L d S}\right)_{e 1}\left(Y_{Q d S}\right)_{11}\left(Y_{L Q S}\right)_{1 e} \mu_{S S S}}{m_{S_{3,2,1 / 6}}^{2} m_{S_{3,1,-1 / 3}}^{2} m_{S_{1,2,1 / 2}}^{2}} \frac{1}{16}\left(\mathcal{O}_{1}^{S R}\right)_{\{R R\} R}
\end{aligned}
$$

The experimental bound, see eq. (2.7), on $0 \nu \beta \beta$ decay can then be interpreted again as an upper limit on the new leptonic Yukawa interactions as:

$$
\left(Y_{L d S}\right)_{e 1}\left(Y_{L Q S}\right)_{1 e}<0.15\left(\frac{m_{S_{3,2,1 / 6}}^{2} m_{S_{3,1,-1 / 3}}^{2} m_{S_{1,2,1 / 2}}^{2}}{1.0\left[\mathrm{TeV}^{6}\right]}\right)\left(\frac{1.0[\mathrm{TeV}]}{\mu_{S S S}}\right)\left(\frac{1.0}{\left(Y_{Q d S}\right)_{11}}\right)
$$

\footnotetext{
${ }^{5}$ There are also three more non-genuine diagrams, discussed in [77], which can be understood as one-loop generated vertices for one of the three tree-level seesaws.

${ }^{6}$ The remaining diagram $\mathrm{T} \nu$-1-i can be understood as opening-up of the quartic scalar vertex in $\mathrm{T} \nu-3$, by inserting an additional scalar.
} 
With the interactions shown in eq. (2.13), neutrinos acquire Majorana masses through the diagram $\mathbf{T} \nu$-1-ii. This neutrino mass generation mechanism through the leptoquarkHiggs coupling was first proposed in [79] and discussed in detail in [40, 80].

Assuming the coupling $\mu_{S S S}$ is smaller than the average of the leptoquark quark masses $m_{\mathrm{LQ}}=\left(m_{S_{3,2,1 / 6}}+m_{S_{3,1,-1 / 3}}\right) / 2$, we can roughly estimate the neutrino mass as

$$
\left(m_{\nu}\right)_{\alpha \beta} \simeq \frac{1}{16 \pi^{2}} \frac{m_{d_{k}} \mu_{S S S}}{m_{\mathrm{LQ}}^{2}}\left\langle S_{1 / 2}\right\rangle\left[\left(Y_{L Q S}^{\dagger}\right)_{\beta k}\left(Y_{L d S}^{\dagger}\right)_{k \alpha}+(\beta \leftrightarrow \alpha)\right]
$$

where $m_{d_{k}}$ is the mass of the down-type quark (of generation $k$ ), which enters this estimation as the vertex at the left-upper corner of $\mathbf{T} \nu$-1-ii of figure 4. Applying the bound on the effective Majorana mass $\left\langle m_{\nu}\right\rangle<0.3 \mathrm{eV}$ (which is obtained from eq. (2.7) with the assumption of the mass mechanism dominance) to eq. (2.16), we have

$$
\left(Y_{L d S}\right)_{e k}\left(Y_{L Q S}\right)_{e k}<0.034\left(\frac{4.0[\mathrm{GeV}]}{m_{d_{k}}}\right)\left(\frac{m_{\mathrm{LQ}}}{1.0[\mathrm{TeV}]}\right)^{2}\left(\frac{1.0[\mathrm{MeV}]}{\mu_{S S S}}\right)\left(\frac{174[\mathrm{GeV}]}{\left\langle S_{1 / 2}\right\rangle}\right)\left(\frac{\left\langle m_{\nu}\right\rangle}{0.3[\mathrm{eV}]}\right) .
$$

Assuming that the flavour structure of the new Yukawa interactions in eq. (2.13) is not strongly hierarchical, one concludes that third generation quarks give the largest contribution to the neutrino mass. If the vev $\left\langle S_{1 / 2}\right\rangle$ is as large as the SM Higgs vev, this constraint is more than six (three) orders of magnitude more stringent than eq. (2.15) for $k=3$ $(k=1)$. Note again that if the vev $\left\langle S_{1 / 2}\right\rangle$ vanishes then, as in the tree-level case, the $d=9$ contribution to $0 \nu \beta \beta$ and the mass mechanism in this class of models are independent of each other.

If the leptoquark mass is set to $\mathcal{O}(1) \mathrm{TeV}$ and Yukawa couplings are taken to be $\mathcal{O}(0.1)$, the trilinear coupling $\mu_{S S S}$ must be $\mathcal{O}(100) \mathrm{keV}$ to reproduce $\mathcal{O}(0.05) \mathrm{eV}$ of neutrino masses, which is the minimum value necessary to reproduce data on atmospheric neutrino oscillations. Such a small value of the coupling $\mu_{S S S}$ can (obviously) be probed only in a $0 \nu \beta \beta$ process dominated by the mass mechanism, since there is no other LNV process, for which experiments have even remotely comparable sensitivity.

To finish this discussion of the one-loop neutrino mass case, recall the possible two-loop contributions to neutrino masses in this model. As shown in the next subsection (and the list in the appendix), the model described with eq. (2.13) generates neutrino masses at the two-loop level, even if the value of $\left\langle S_{1 / 2}\right\rangle$ is identical with zero. However, the one-loop contribution discussed in this subsection can easily (even with a value of $\left\langle S_{1 / 2}\right\rangle$ smaller than $\mathrm{MeV}$ ) dominate over the two-loop diagrams, as can be seen from eqs. (2.16)-(2.17). Therefore, we classify this type of the models separately from the genuine two-loop models, which will be defined and explained in detail in the next subsection.

Very similar arguments can be applied to the other two topologies, $\mathbf{T} \nu \mathbf{- 1}$-iii and $\mathbf{T} \nu \mathbf{- 3}$, shown in figure 4. Such one-loop neutrino mass models appear in quite a large number of decompositions. We give the complete list of this class of models in table 2 in the appendix, together with the additional interaction that is required (and is allowed by the SM gauge symmetries) to generate the corresponding one-loop diagram. 


\subsection{2-loop models}

The effective operators $\mathcal{O}_{11}, \mathcal{O}_{12}$, and $\mathcal{O}_{14}$ contain two lepton doublets, and thus naively one expects them to generate neutrino masses at 2 -loop level, ${ }^{7}$ when their quark legs are connected with the SM Yukawa interactions. However, this naive picture has to be modified, once the possible decompositions of the effective operators are taken into account.

In this subsection, we will first demonstrate why some of the decompositions of the operators $\mathcal{O}_{11}, \mathcal{O}_{12}$, and $\mathcal{O}_{14}$ do not generate neutrino masses genuinely at the 2-loop level. Here, we use the terminology genuine 2-loop diagrams, following [62], to imply that the corresponding model (or decomposition in our case) generates neutrino masses at 2-loop order and that no lower order diagram exists.

Let us first discuss the decomposition of $\mathcal{O}_{14}$. This operator contains:

$$
\bar{L}_{\dot{a}}, \quad \bar{L}_{\dot{a}}, \quad{\overline{u_{R}}}^{a}, \quad Q_{a}, \quad \bar{Q}_{\dot{a}}, \quad d_{R}{ }^{\dot{a}} .
$$

Here we explicitly wrote the 2-component spinor indices for the fermions: dotted for a right-handed field (complex conjugate of left-handed field), undotted for a left-handed field. As everywhere else in this paper, we restrict the discussion to fermions and scalars as mediators. For $\mathcal{O}_{14}$ this implies that the spinor indices on $Q$ and $\overline{u_{R}}$ must be contracted for the effective operator being a Lorentz scalar. There are only two choices to assign these two quarks to the outer legs of a $d=9$ tree diagram: (i) They form a Yukawa interaction with a scalar mediator, i.e., $\left(\overline{u_{R}} Q \cdot S\right)$. This is shown as the upper diagram of figure 5 . Or: (ii) Each of these quarks forms a Yukawa interaction with a fermion mediator $\psi$ and one of the scalar mediators, i.e., $\left(\overline{u_{R}} \psi S\right)\left(\bar{\psi} Q S^{\prime}\right)$. This is shown as the lower diagram of figure 5. When the loops are closed, as shown on the right of figure 5, via the SM Yukawa interaction $y_{u}\left(\bar{Q} \cdot H^{*} u_{R}\right)$, the resulting quark loop is divergent. In other words, these loops are infinite corrections for (i) a mass term mixing the scalar mediator and the SM Higgs doublet $m_{S H}^{2} S H^{*}$, or (ii) a corresponding term mixing two scalar mediators $m_{S S^{\prime}}^{2} S S^{\prime}$. Therefore, the original tree-level Lagrangian generating these operators must contain these scalar mass terms as counter terms for the infinities, and the quark loop appearing in neutrino mass diagrams must be substituted with those scalar mass terms.

This simple argument actually holds for any decomposition of $\mathcal{O}_{14}$. In short, one cannot construct a genuine (irreducible) 2-loop diagram for neutrino masses from the decomposition of $\mathcal{O}_{14}$, if the mediators are restricted to scalars and fermions. For this reason, in the appendix the decompositions of $\mathcal{O}_{14}$ are instead either listed under "treelevel" or "1-loop", depending on the additional interaction necessary.

The next question we must address is: can all the decompositions of $\mathcal{O}_{11(b)}$ and $\mathcal{O}_{12(a)}$ genuinely generate neutrino masses at 2-loop level? The answer is no, and the argument for those cases is very similar to the one presented above for $\mathcal{O}_{14}$. Two concrete examples are shown in figure 6 . Consider first the diagram on the left, based on decomposition T-I-1-i

\footnotetext{
${ }^{7}$ When the two lepton doublets are anti-symmetric in $\mathrm{SU}(2)_{L}$, an additional loop is necessary to obtain a neutrino mass term, i.e., the resulting neutrino mass diagram contains three loops, which correspond to $\mathcal{O}_{11 a}, \mathcal{O}_{12 b}$ and $\mathcal{O}_{14 a}$ in the list of [36]. In $0 \nu \beta \beta$ decay only the operators $\mathcal{O}_{11 b}, \mathcal{O}_{12 a}$ and $\mathcal{O}_{14 b}$, which contain symmetric pieces in $\mathrm{SU}(2)_{L}$, can appear. These are called $\mathcal{O}_{11}, \mathcal{O}_{12}$ and $\mathcal{O}_{14}$ for brevity here and in [31].
} 

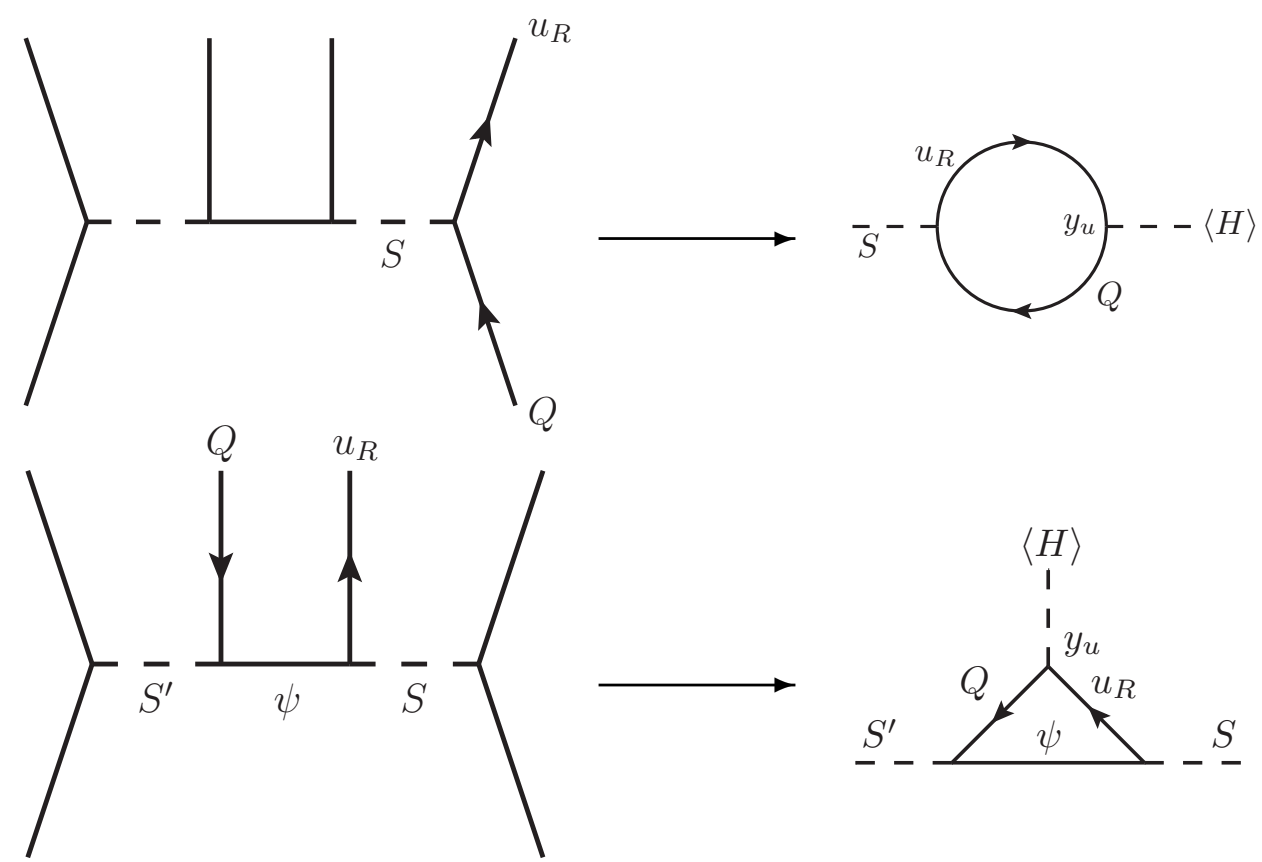

Figure 5. Decompositions of the Babu-Leung operator \#14, $\mathcal{O}_{14} \propto \bar{L} \bar{L} \bar{Q} d_{R} \overline{u_{R}} Q$, with two scalar $\left(S\right.$ and $\left.S^{\prime}\right)$ and one fermion $(\psi)$ mediators. For a neutrino mass diagram the quark legs $\overline{u_{R}}$ and $Q$ must be connected via the SM Yukawa interaction $y_{u}$. The resulting loops (shown on the right) are infinite one-loop corrections to the scalar mass terms, that therefore must be contained in the tree-level Lagrangians of the corresponding models.

$\mathcal{O}_{12}$. The inner loop in these classes of neutrino mass diagrams actually corresponds to an infinite (1-loop) correction to the scalar quartic interaction $\lambda_{S S^{\prime} H H} S S^{\prime} H H$. In the diagram on the right, the "inner" loop involving $S$ generates a Yukawa interaction $y_{Q \psi H} \bar{\psi} Q \cdot H$. Again, this correction is infinite, thus requiring a tree-level counter term which must be contained in the original Lagrangian. Given these additional (but required) interactions, the models contain neutrino mass diagrams at 1-loop order. Note that, the left diagram in figure 6 corresponds to Diagram (A) of figure 14 in [37] (with appropriate Higgs insertions) and also Diagram (c) of figure 5 in [81]. The right diagram is Diagram (B) in [37] and (d) in [81]. Quite a number of possible decompositions of $\mathcal{O}_{11}$ and $\mathcal{O}_{12}$ follow this pattern and are thus listed in the tables in the appendix as 1-loop models, together with the additional-but-necessary interactions.

After filtering out all decompositions that result in non-genuine 2-loop neutrino mass diagrams, we have found that for all remaining decompositions there are only three types of genuine 2-loop diagrams, all of them based on $\mathcal{O}_{11}$. These are shown in figure 7 . The naming scheme in this figure follows [62]. In the appendix, we present the complete list of the genuine 2-loop neutrino mass models and specify the class of neutrino mass diagrams, into which each model falls. In table 4, two of the decompositions based on the BL operator $\mathcal{O}_{19}$ are also listed. These appear in the table for two-loop models due to the fact that the intermediate fermion is of Majorana type, i.e., for these decompositions, the "asymmetric" op- 

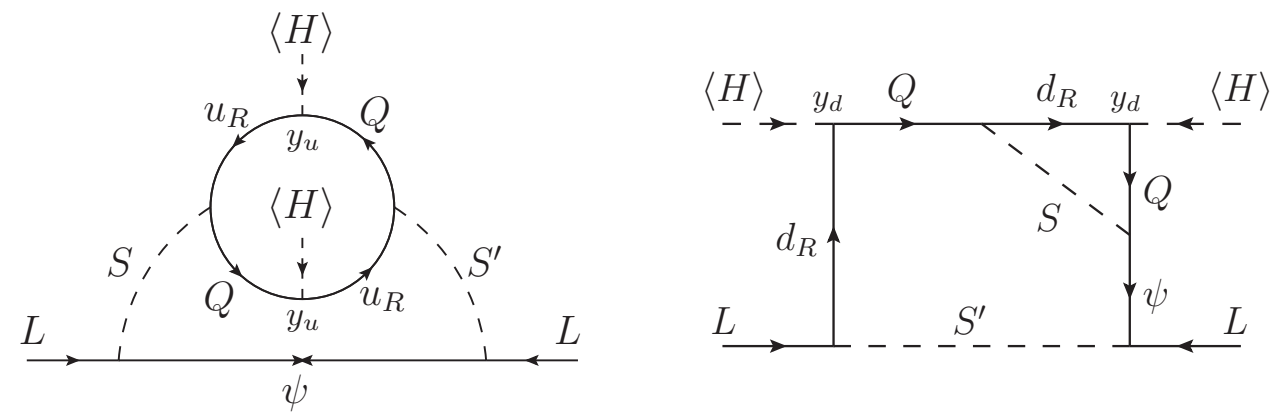

Figure 6. Examples of non-genuine 2-loop neutrino mass diagrams, based on the decompositions of T-I-1-i $\mathcal{O}_{12}$ (left) and T-I-2-ii-a $\mathcal{O}_{11}$ (right). For a discussion see text.
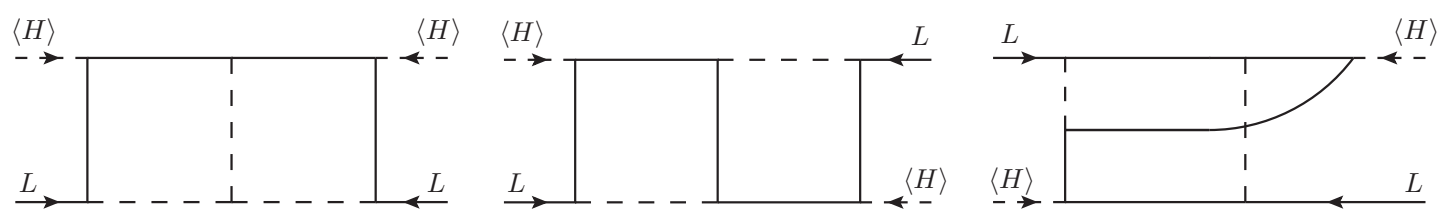

Figure 7. Genuine 2-loop neutrino mass diagrams based on decomposition of the Babu-Leung operator $\mathcal{O}_{11}$. From left to right the diagrams are identified as CLBZ-1, PTBM-1 and PTBM-4, following the classification of [62]. The left diagram (CLBZ-1) was discussed in the context of a neutrino mass model in [19], and corresponds to the diagram in figure 10 in [37] and (e) in [81]. The diagram (PTBM-1) in the middle was discussed in [82], and corresponds to Diagram (D2) in figure 14 in [37] and (f) in figure 5 in [81]. The diagram on the right corresponds to Diagram (C) in [37] and (f) in figure 5 [81]. A model based on this diagram will be discussed in section 3 .

erator $\mathcal{O}_{19} \propto \bar{L} \overline{e_{R}}$ is always accompanied by the "symmetric" operator $\mathcal{O}_{11} \propto \bar{L} \bar{L}$, and the associated operator generates neutrino masses at the two-loop level. The catalogue of the effective operators appearing with their associated operators is given in the tables of [31].

Among these three genuine diagrams, neutrino mass models based on the CLBZ-1 and the PTBM-1 diagrams have already been studied in the context of the decomposition of the $d=9$ operators $[19,82]$. Therefore, we will discuss a 2-loop neutrino mass model that is associated with the remaining possibility, i.e., the PTBM-4 diagram. Here, as in the previous subsections, we compare the $d=9$ contribution to $0 \nu \beta \beta$ with the mass mechanism contribution and postpone the detailed discussion on phenomenology of this model till section 3 .

The example we choose is based on T-I-4-ii-b, $\mathcal{O}_{11}$. The Lagrangian for this decomposition contains the terms

$$
\begin{aligned}
\mathcal{L}= & \left(Y_{Q Q S}\right)_{i j}\left(\bar{Q}_{i} \vec{\tau} \cdot Q_{j}^{c}\right) \hat{\vec{S}}_{6,3,1 / 3}+\left(Y_{L \psi S}\right)_{\alpha}\left(\bar{L}_{\alpha} \vec{\tau} \psi_{6,2,1 / 6}\right) \vec{S}_{6,3,1 / 3}^{\dagger} \\
& +\left(Y_{\psi d S}\right)_{i}\left(\overline{\hat{\psi}}_{6,2,1 / 6} d_{R, i}\right) S_{3,2,1 / 6}+\left(Y_{L d S}\right)_{\alpha i}\left(\bar{L}_{\alpha} d_{R, i}\right) \cdot S_{3,2,1 / 6}^{\dagger}
\end{aligned}
$$

We use the notation $\hat{\vec{S}}_{6,3,1 / 3}=\left(\vec{S}_{6,3,1 / 3}\right)_{X}\left(T_{\overline{\mathbf{6}}}\right)_{I J}^{X}$ and $\hat{\psi}_{6,2,1 / 6}=\left(\psi_{6,2,1 / 6}\right)_{X}\left(T_{\overline{\mathbf{6}}}\right)_{I J}^{X}$. The tensors $T_{\mathbf{6}}$ and $T_{\overline{\mathbf{6}}}$ in the $\mathrm{SU}(3)_{c}$ are given in [31]. Here, $\vec{\tau}$ is the Pauli matrix vector for a triplet of $\mathrm{SU}(2)_{L}$. The effective $d=9$ operator resulting from this Lagrangian can 
be written with the following linear combination of the basis operators $\mathcal{O}_{i \in\{1-5\}}^{S R}$ of the short-range contributions to $0 \nu \beta \beta$ decay as:

$$
\begin{aligned}
\mathcal{L}_{\text {eff }} & =-\frac{\left(Y_{Q Q S}\right)_{11}\left(Y_{L \psi S}\right)_{e}\left(Y_{\psi d S}\right)_{1}\left(Y_{L d S}\right)_{e 1}}{m_{S_{6,3,1 / 3}}^{2} m_{S_{3,2,1 / 6}}^{2} m_{\psi}}\left[\left(\bar{Q}_{1} T_{\overline{\mathbf{6}}} \tau^{a} \cdot Q_{1}^{c}\right)\left(\bar{L}_{e} \tau^{a}\right)\left(d_{R} T_{\mathbf{6}}\right) \cdot\left(L_{e}{ }^{c} d_{R}\right)\right]+\text { H.c. } \\
& \supset \frac{\left(Y_{Q Q S}\right)_{11}\left(Y_{L \psi S}\right)_{e}\left(Y_{\psi d S}\right)_{1}\left(Y_{L d S}\right)_{e 1}}{m_{S_{6,3,1 / 3}}^{2} m_{S_{3,2,1 / 6}}^{2} m_{\psi}}\left[\frac{1}{16}\left(\mathcal{O}_{1}^{S R}\right)_{\{R R\} R}-\frac{1}{64}\left(\mathcal{O}_{2}^{S R}\right)_{\{R R\} R}\right]
\end{aligned}
$$

and the experimental bound eq. (2.7) constrains a combination of the coefficients

$$
\left(Y_{Q Q S}\right)_{11}\left(Y_{L \psi S}\right)_{e}\left(Y_{\psi d S}\right)_{1}\left(Y_{L d S}\right)_{e 1}<6.3 \cdot 10^{-3}\left(\frac{m_{S_{3,2,1 / 6}}^{2} m_{S_{6,3,1 / 3}}^{2} m_{\psi}}{1.0\left[\mathrm{TeV}^{5}\right]}\right)
$$

On the other hand, the neutrino mass generated from the 2-loop diagram based on the effective operator eq. (2.20) also contributes to $0 \nu \beta \beta$ through the mass mechanism. The size of 2-loop neutrino mass diagram can be roughly estimated as [35-37, 62]

$$
\left(m_{\nu}\right)_{\alpha \beta} \simeq \frac{N_{c}}{\left(16 \pi^{2}\right)^{2}} \frac{m_{b}^{2}}{\Lambda_{\mathrm{LNV}}}\left[\left(Y_{Q Q S}\right)_{33}\left(Y_{L \psi S}\right)_{\alpha}\left(Y_{\psi d S}\right)_{3}\left(Y_{L d S}\right)_{\beta 3}+(\beta \leftrightarrow \alpha)\right] .
$$

Applying the experimental bound from $0 \nu \beta \beta$ to eq. (2.22) and substituting $N_{c}=6$ because of the colour sextet combination in the loop, we can place the bound on the couplings of the third generation quarks:

$$
\left(Y_{Q Q S}\right)_{33}\left(Y_{L \psi S}\right)_{e}\left(Y_{\psi d S}\right)_{3}\left(Y_{L d S}\right)_{e 3}<7.2 \cdot 10^{-5}\left(\frac{4.18[\mathrm{GeV}]}{m_{b}}\right)^{2}\left(\frac{\Lambda_{\mathrm{LNV}}}{1.0[\mathrm{TeV}]}\right)\left(\frac{\left\langle m_{\nu}\right\rangle}{0.3[\mathrm{eV}]}\right)
$$

As this rough estimation shows, the mass mechanism and the short-range part of the amplitude give similar contributions to $0 \nu \beta \beta$ decay. Note that assuming flavour democratic Yukawa couplings, the constraints eq. (2.21) and eq. (2.23) become equally strong if the mass scale of the new particles is taken to be roughly $\sim 300 \mathrm{GeV}$. For larger mass values, the short range contribution can dominate only if Yukawas with index " 3 " are smaller than those with index "1", otherwise the mass mechanism dominates. A more detailed discussion using the full expression for the two-loop neutrino mass integral will be presented in section 3 .

\section{$2.5 \quad 3$-loop models}

From the point-of-view of effective operators, the Babu-Leung operators $\mathcal{O}_{19}$ and $\mathcal{O}_{20}$ require three SM Yukawa interactions to generate neutrino masses: two quark Yukawa interactions and one charged-lepton Yukawa interaction, to convert $e_{R}$ in the effective operators to $L$ for a neutrino mass. This fact leads us to three-loop neutrino mass models. However, some of the possible decompositions of $\mathcal{O}_{19}$ and $\mathcal{O}_{20}$ contain the ingredients to generate neutrino masses at a level lower than three-loop. In such a case, the lower loop contributions can easily dominate neutrino masses and make the contribution from a three-loop diagram sub-dominant. This can happen for two reasons. First, there are 


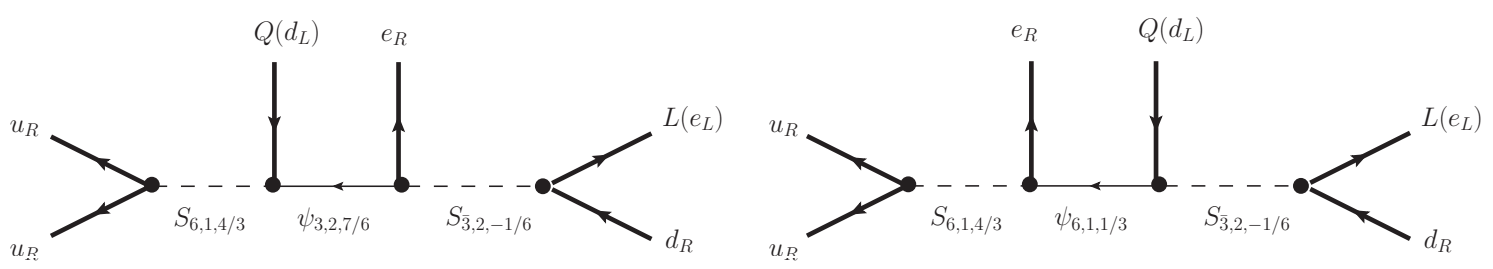

Figure 8. The Feynman diagrams for the decompositions T-I-4-ii-a (left) and T-I-4-ii-b (right) for BL operator $\mathcal{O}_{20}$. The former leads to 2-loop $d=7$ neutrino masses, while the latter is an example of a 3-loop neutrino mass model, see text.
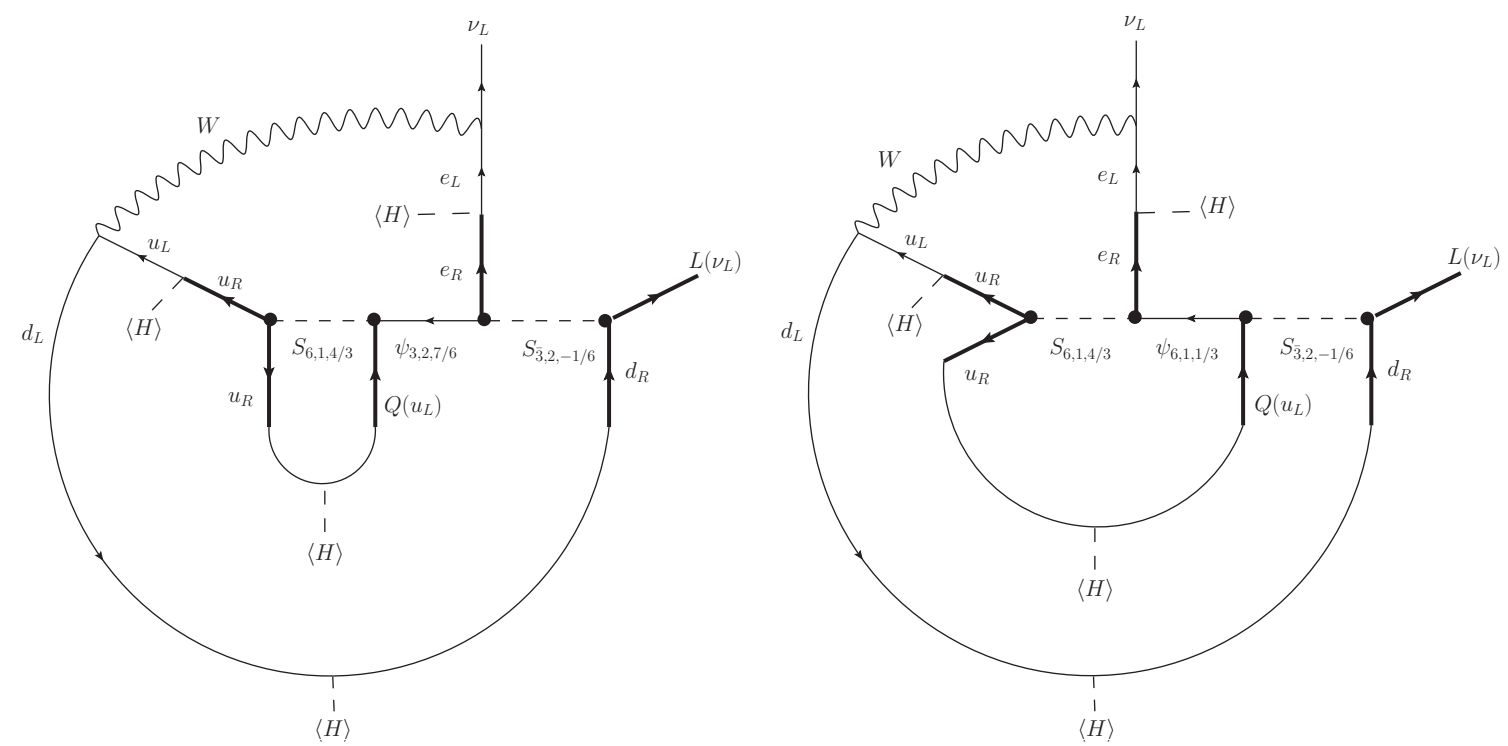

Figure 9. Examples of 3-loop diagrams for the decompositions T-I-4-ii-a (left) and T-I-4-ii-b (right) for BL operator $\mathcal{O}_{20}$, see figure 8. The diagram to the right corresponds to a genuine 3-loop model, while the one to the left is not. See text.

decompositions based on $\mathcal{O}_{19}$ or $\mathcal{O}_{20}$, in which $\mathcal{O}_{11}$ necessarily also appears. We call this "associated" operators and classify those decompositions in the class corresponding to those lower loop levels, which usually will dominate over the 3-loop contribution. Decompositions T-I-2-iii-a and T-I-5-i of $\mathcal{O}_{19}$ with the Majorana fermion $\psi_{8,1,0}$, which are listed in the table of two-loop models (table 4), are categorised in this class. And, second, there are decompositions for $\mathcal{O}_{19}$ or $\mathcal{O}_{20}$, in which the 3-loop diagrams are not genuine in the sense that one of the sub-diagrams corresponds to the 1-loop generation of a certain vertex. We will discuss this case in a bit more detail.

Take the examples of the decompositions T-I-4-ii-a and T-I-4-ii-b, both $\mathcal{O}_{20}$. The Feynman diagrams are given in figure 8, and show T-I-4-ii-a $\rightarrow\left(\overline{u_{R}} \overline{u_{R}}\right)\left(d_{L}\right)\left(\overline{e_{R}}\right)\left(\overline{e_{L}} d_{R}\right)$ and T-I-4-ii-b $\rightarrow\left(\overline{u_{R}} \overline{u_{R}}\right)\left(\overline{e_{R}}\right)\left(d_{L}\right)\left(\overline{e_{L}} d_{R}\right)$ graphically. As we will see, despite the similarity between these two cases, T-I-4-ii-b will lead to a genuine 3-loop model, while T-I-4-ii-a will not. Consider the examples of 3-loop diagrams for these two decompositions shown in figure 9. First of all, note that the loop diagrams shown in figure 9 should be understood 


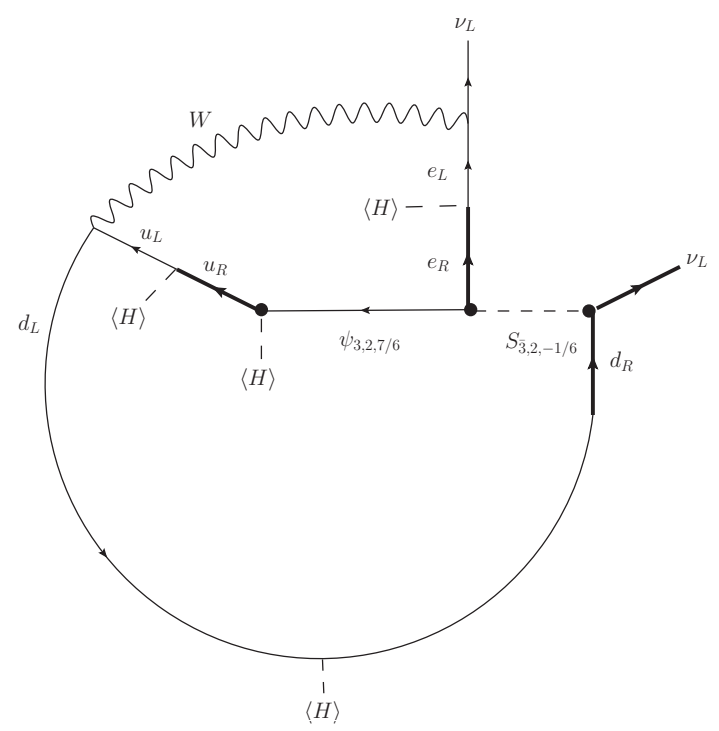

Figure 10. Example of a 2-loop $d=7$ diagram for the decomposition T-I-4-ii-a. This diagram corresponds to Diagram (a) in figure 18.

as examples only, because there might be more than one diagram contributing to the full neutrino mass matrix for each decomposition. In the diagram for T-I-4-ii-a (left), one sees that the innermost loop effectively generates the vertex $\overline{u_{R}} \psi_{3,2,7 / 6} H^{\dagger}$ at 1-loop order. This one-loop sub-diagram is infinite and, therefore, a tree-level counter term is necessarily to be included in the Lagrangian to absorb the infinity. In fact, the quantum numbers of the particles involved in the loop are such that the necessary vertex actually cannot be forbidden at tree-level by the SM gauge symmetry. This tree-level coupling has a value that is not fixed by the $0 \nu \beta \beta$ decay amplitude, but the 2-loop $(d=7)$ diagram that results from this coupling, see figure 10 , can easily dominate over the 3 -loop diagram. ${ }^{8}$ We have classified therefore all these cases of $\mathcal{O}_{19}$ and $\mathcal{O}_{20}$, where such a tree-level vertex is allowed, as "2-loop $d=7$ " (table 5 ) in appendix. The loop diagram based on the decomposition T-I-4-ii-b, which is shown as the right diagram of figure 9, does not contain such an inner loop and, thus, such a construction is not possible for this decomposition. Decompositions of this type are therefore classified as genuine 3-loop models in the appendix.

We give here a rough estimate of the size of the neutrino mass generated by the 3-loop diagram based on T-I-4-ii-b in order to present some general arguments on the relative size of the $d=9$ contributions and the mass mechanism contributions to $0 \nu \beta \beta$ in this class of models. This example, in which the $d=9$ is mediated by a di-quark, $S_{6,1,4 / 3}$, a leptoquark, $S_{3,2,1 / 6}$, and an exotic colour-sextet fermion, $\psi_{6,1,1 / 3}$, leads to a Lagrangian that contains the terms:

$$
\begin{aligned}
\mathcal{L}= & \left(Y_{u u S}\right)_{i j} \overline{u_{R, i}} \hat{S}_{6,1,4 / 3} u_{R, j}{ }^{c}+\left(Y_{e \psi S}\right)_{i} \overline{\psi_{6,1,1 / 3}} e_{R, i} S_{6,1,4 / 3} \\
& +\left(Y_{Q \psi S}\right)_{i} \overline{Q_{i}} \hat{\psi}_{6,1,1 / 3} \cdot\left(S_{3,2,1 / 6}\right)^{\dagger}+\left(Y_{L d S}\right)_{i j} \overline{d_{R, j}} L_{i} \cdot S_{3,2,1 / 6}+\text { H.c.. }
\end{aligned}
$$

\footnotetext{
${ }^{8}$ Naively, the 2-loop $d=7$ contribution becomes more important than the 3-loop contribution, when the tree-level coupling $Y_{u \psi_{H}}$ of the necessary interaction $\overline{u_{R}} \psi_{3,2,7 / 6} H^{\dagger}$ is larger than $Y_{u u S} Y_{Q \psi S} /\left(16 \pi^{2}\right) \times($ some logarithmic factor).
} 
As above, we use the notation $\hat{S}_{6,1,4 / 3}=\left(S_{6,1,4 / 3}\right)_{X}\left(T_{\overline{\mathbf{6}}}\right)_{I J}^{X}$ and $\hat{\psi}_{6,1,1 / 3}=\left(\psi_{6,1,1 / 3}\right)_{X}\left(T_{\overline{\mathbf{6}}}\right)_{I J}^{X}$. Together, the $Y_{e \psi S}, Y_{Q \psi S}$, and $Y_{L d S}$ terms necessarily violate lepton number by two units. All generation indices in the couplings in eq. (2.24) have been suppressed for simplicity. The contribution to the neutrino mass matrix can be roughly estimated as

$$
\left(m_{\nu}\right)_{\alpha \beta} \simeq \frac{N_{c}}{\left(16 \pi^{2}\right)^{3}}\left[\frac{m_{t}^{2} m_{b} m_{e_{\alpha}}}{\Lambda_{\mathrm{LNV}}^{3}}\left(Y_{u u S}\right)_{33}\left(Y_{e \psi S}\right)_{\alpha}\left(Y_{Q \psi S}\right)_{3}\left(Y_{L d S}\right)_{3 \beta}+(\alpha \leftrightarrow \beta)\right],
$$

where $\Lambda_{\mathrm{LNV}} \simeq m_{S_{6,1,4 / 3}} \simeq m_{S_{3,2,1 / 6}} \simeq m_{\psi}$ is the mass scale of the heavy states, which is typically taken to be TeV. $N_{c}$ is a colour factor. Here, we assumed that all the SM fermion masses are much smaller than $\Lambda_{\mathrm{LNV}}$. Putting all the Yukawa couplings in eq. (2.25) equal to unity and $\Lambda_{\mathrm{LNV}}=1 \mathrm{TeV}$ and $N_{c}=6$ (for a colour sextet combination), one finds ${ }^{9}$

$$
\left(m_{\nu}\right)_{e e} \sim 1 \times 10^{-5} \mathrm{eV},\left(m_{\nu}\right)_{\mu \mu} \sim 2 \times 10^{-2} \mathrm{eV},\left(m_{\nu}\right)_{\tau \tau} \sim 0.3 \mathrm{eV} .
$$

This implies that the mass mechanism contribution to $0 \nu \beta \beta$ is guaranteed to be subdominant in this class of models. Also, eq. (2.26) shows that 3-loop models can potentially explain neutrino oscillation data only if all of the involved Yukawa couplings are set to be $\mathcal{O}(1)$. Thus, we expect such models to be quite constrained from upper limits on flavour violating decays of charged leptons. We will not discuss this class of models in more detail here, since their detailed phenomenology is outside the scope of this paper.

The effective $d=9$ operator resulting from the Lagrangian eq. (2.24) can be written with the following linear combination of the basis operators $\mathcal{O}_{i \in\{1-5\}}^{S R}$ of the short-range contributions to $0 \nu \beta \beta$ decay as:

$$
\mathcal{L}_{\text {eff }}=\frac{\left(Y_{u u S}\right)_{11}\left(Y_{e \psi S}\right)_{e}\left(Y_{Q \psi S}\right)_{1}\left(Y_{L d S}\right)_{e 1}}{m_{S_{1 / 3}}^{2} m_{S_{1 / 6}}^{2} m_{\psi}}\left[\frac{1}{16 i}\left(\mathcal{O}_{4}^{S R}\right)_{\{R R\} R}-\frac{1}{16}\left(\mathcal{O}_{5}\right)_{\{R R\} R}^{S R}\right]
$$

and the experimental bound eq. (2.7) constrains a combination of the coefficients to be:

$$
\left(Y_{u u S}\right)_{11}\left(Y_{e \psi S}\right)_{e}\left(Y_{Q \psi S}\right)_{1}\left(Y_{L d S}\right)_{e 1}<1.5 \cdot 10^{-2}\left(\frac{m_{S_{1 / 6}}^{2} m_{S_{1 / 3}}^{2} m_{\psi}}{1.0\left[\mathrm{TeV}^{5}\right]}\right) .
$$

The difference in the short-range bounds, eq. (2.21) and eq. (2.28), is due to the different values of nuclear matrix elements entering the transition operator. All other three-loop models will have constraints similar to the ones discussed here. They are listed in table 6 in the appendix.

\subsection{4-loop models}

Finally, all operators $\mathcal{O}_{-}=\overline{e_{R}} \overline{e_{R}} \overline{u_{R}} d_{R} \overline{u_{R}} d_{R}$, with exception of decomposition T-I-5-i (see table 1 in the appendix), will lead to four-loop neutrino mass models. The simplest

\footnotetext{
${ }^{9}$ Using $\left\langle m_{\nu}\right\rangle \leq 0.3 \mathrm{eV}$, we can formally write the constraint on the Yukawa couplings in the form of:

$$
\left(Y_{u u S}\right)_{33}\left(Y_{e \psi S}\right)_{e}\left(Y_{Q \psi S}\right)_{3}\left(Y_{L d S}\right)_{e 3}<3 \cdot 10^{3}\left(\frac{\Lambda_{\mathrm{LNV}}^{3}}{1.0\left[\mathrm{TeV}^{3}\right]}\right),
$$
}

which is much worse than even the trivial constraint derived from perturbativity. 

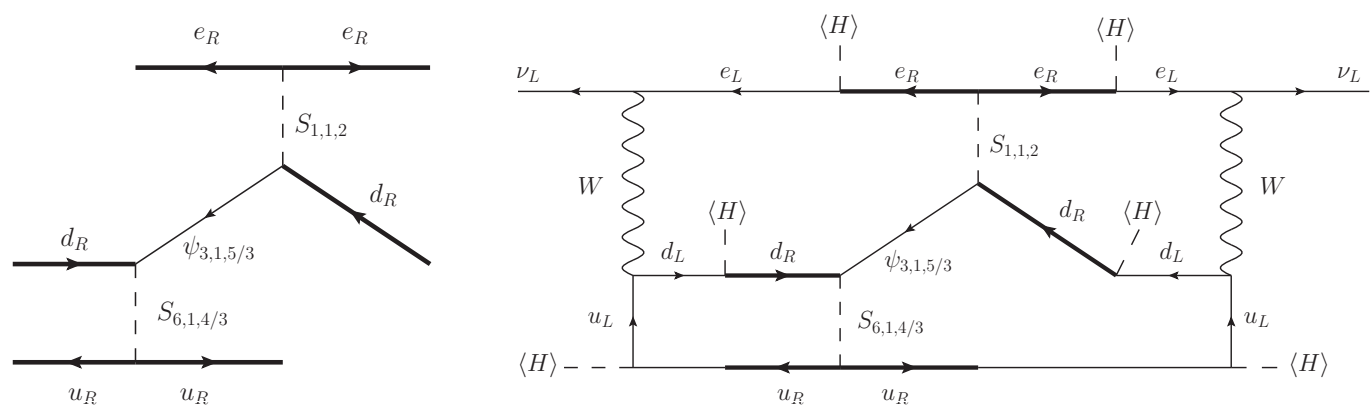

Figure 11. An example of a four-loop neutrino mass model. To the left: $0 \nu \beta \beta$ decay via the $d=9$ operator $\left(\overline{u_{R}} \overline{u_{R}}\right)\left(d_{R}\right)\left(d_{R}\right)\left(\overline{e_{R}} \overline{e_{R}}\right)$. To the right: four-loop $d=9$ neutrino mass, see text.

possibility to construct a four-loop diagram for these operators is to use a SM charged current interaction. We estimate that this gives the dominant contribution to the neutrino mass. Here we show an example of the decompositions of the $0 \nu \beta \beta$ decay operator $\mathcal{O}_{-}$in figure 11, which is based on decomposition T-I-3-ii $\left(\overline{u_{R}} \overline{u_{R}}\right)\left(d_{R}\right)\left(d_{R}\right)\left(\overline{e_{R}} \overline{e_{R}}\right)$. The four-loop neutrino mass diagram based on this decomposition is also shown on the right. Taking the limit $m_{\psi_{3,1,5 / 3}} \sim m_{S_{6,1,4 / 3}} \sim m_{S_{1,1,2}} \gg m_{W}, m_{t}$ one can estimate the order of magnitude of this four-loop diagram, which is,

$$
\left(m_{\nu}\right)_{\alpha \beta} \sim \frac{g^{4}}{\left(16 \pi^{2}\right)^{4}} \frac{m_{e_{\alpha}} m_{e_{\beta}} m_{u_{i}} m_{u_{j}} m_{d_{i}} m_{d_{j}}}{m_{\psi_{3,1,5 / 3}} m_{S_{6,1,4 / 3}}^{2} m_{S_{1,1,2}}^{2}}\left(Y_{\psi d S}\right)_{i}\left(Y_{e e S}\right)_{\alpha \beta}\left(Y_{u u S}\right)_{i j}\left(Y_{\psi d S}\right)_{j} .
$$

The expression eq. (2.29) shows that this four-loop contribution would yield only $\left(m_{\nu}\right)_{\tau \tau} \sim$ $\mathcal{O}\left(10^{-10}\right) \mathrm{eV}$ for $m_{\psi_{3,1,5 / 3}} \sim m_{S_{6,1,4 / 3}} \sim m_{S_{1,1,2}} \sim 1 \mathrm{TeV}$, even when choosing all SM fermion masses to be third generation. Since this is obviously many orders of magnitude below the values of neutrino masses required from oscillation experiments, models of this category by themselves cannot be considered realistic. Of course, neutrinos could be quasi-Dirac particles, explaining oscillation data by Dirac mass terms (using additionally introduced right-handed neutrinos), while $0 \nu \beta \beta$ decay is dominated by the short-range diagrams such as the one shown in figure 11. However, constraints on Yukawa couplings will be similar to those derived in the previous subsections in eq. (2.20) and eq. (2.27), with the exact value depending on the decomposition under consideration. All four-loop cases are listed in table 7 in the appendix.

\section{A concrete 2-loop example}

In this section we will discuss one concrete genuine 2-loop neutrino mass model in some more detail. The example we choose is based on the decomposition T-I-4-ii-b of the BabuLeung operator $\mathcal{O}_{11}$, which has not been discussed in the literature before. However, all $0 \nu \beta \beta$ decompositions that generate 2-loop neutrino masses behave quite similarly, in what concerns fits for neutrino oscillation data and constraints from lepton flavour violation searches. Thus, most of the discussion presented below can be applied qualitatively also to all other 2-loop decompositions. 


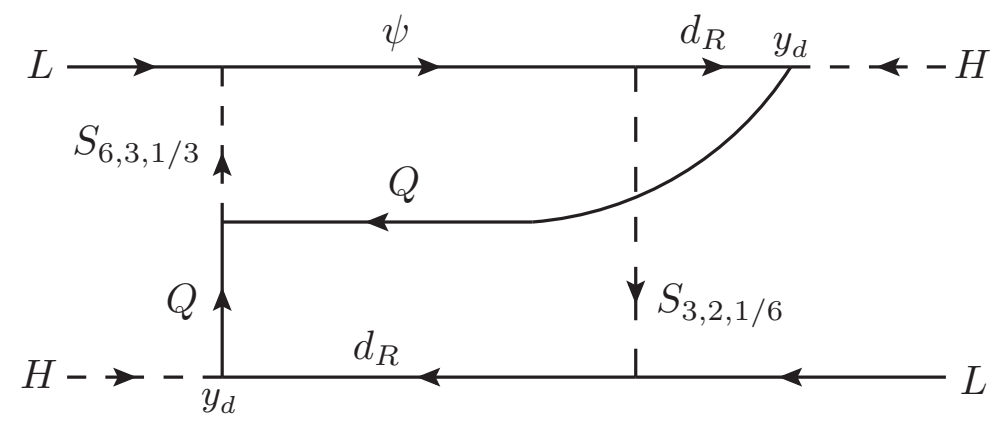

Figure 12. Two loop diagram for neutrino masses generated by the Lagrangian in eq. (3.2).

Any model of neutrino mass must not only generate the correct neutrino mass scale, but also be able to explain the observed neutrino mixing angles. For a recent update of all oscillation data, see, for example, [83]. In addition, since the neutrino mass matrix has a non-trivial flavour pattern, one also expects that low-energy models ${ }^{10}$ of neutrino mass are constrained by charged lepton flavour violation (LFV) searches. Here we will discuss only $\mu \rightarrow e \gamma$, since the experimental upper limit on this process provides usually the most stringent constraints in many models. We note that the authors of [82] present a 2-loop model, which corresponds to the decomposition T-I-5-i and discuss also the constraints from other LFV searches, which we expect are very similar in our example.

Below we will discuss two variations of the model based on T-I-4-ii-b. First (in section 3.2), we introduce only one copy of the exotic fermion $\psi_{6,2,-1 / 6}$ for simplicity. Next (in section 3.3), we will allow to have three copies of these fermions, which allows to fit also quasi-degenerate neutrinos.

\subsection{General formulas for neutrino masses and $\mu \rightarrow e \gamma$}

The Yukawa part of the Lagrangian describing the interactions between the exotic diquark, $S_{6,3,1 / 3}$, the leptoquark, $S_{3,2,1 / 6}$, and the coloured vector-like fermion, $\psi_{6,2,-1 / 6}$ can be written as:

$$
\begin{aligned}
\mathcal{L}= & \left(Y_{Q Q S}\right)_{i j}\left(\bar{Q}_{i} \vec{\tau} \cdot Q_{j}^{c}\right) \hat{\vec{S}}_{6,3,1 / 3}+\left(Y_{L \psi S}\right)_{\alpha k}\left(\bar{L}_{\alpha} \vec{\tau} \psi_{k}\right) \vec{S}_{6,3,1 / 3}^{\dagger} \\
& +\left(Y_{\psi d S}\right)_{k i}\left(\overline{\hat{\psi}}_{k} d_{R, i}\right) S_{3,2,1 / 6}+\left(Y_{L d S}\right)_{\alpha i}\left(\bar{L}_{\alpha} d_{R, i}\right) \cdot S_{3,2,1 / 6}^{\dagger}
\end{aligned}
$$

Here, $i, j$ are generation indices for quarks, we use Greek indices for lepton generations and $k$ runs over the number of copies of $\psi_{6,2,-1 / 6}$. This Lagrangian generates a 2-loop diagram which corresponds to PTBM-4 according to classification by [62], see figure 12. Following the general formulas from [62], the neutrino mass matrix can be expressed as:

$$
\begin{aligned}
\left(m_{\nu}\right)_{\alpha \beta}= & \frac{N_{c} m_{\psi_{k}}}{\left(16 \pi^{2}\right)^{2}}\left[\left(Y_{Q Q S}\right)_{i j}\left(Y_{L \psi S}\right)_{\alpha k}\left(Y_{\psi d S}\right)_{k i}\left(Y_{L d S}\right)_{\beta j}+\left(Y_{Q Q S}\right)_{i j}\left(Y_{L \psi S}\right)_{\beta k}\left(Y_{\psi d S}\right)_{k i}\left(Y_{L d S}\right)_{\alpha j}\right] \\
& \times F\left(m_{\psi_{k}}, m_{S_{3,2,1 / 6}}, m_{d_{i}}, m_{S_{6,3,1 / 3}}, m_{d_{j}}\right)
\end{aligned}
$$

\footnotetext{
${ }^{10}$ By "low-energy" we mean TeV-scale, as in contrast to "high-scale" seesaw models.
} 
where $N_{c}$ is a colour factor, with $N_{c}=6$ for this model. Summation over all flavour indices $i, j, k$ is implied. $F\left(m_{\psi_{k}}, m_{S_{3,2,1 / 6}}, m_{d_{i}}, m_{S_{6,3,1 / 3}}, m_{d_{j}}\right)$ is a loop integral defined as:

$$
\begin{aligned}
& F\left(m_{\psi_{k}}, m_{S_{3,2,1 / 6}}, m_{d_{i}}, m_{S_{6,3,1 / 3}}, m_{d_{j}}\right)=\frac{m_{d_{i}} m_{d_{j}}}{\pi^{4}} \\
& \quad \times \int d^{4} q \int d^{4} k \frac{1}{\left(q^{2}-m_{\psi_{k}}^{2}\right)\left(q^{2}-m_{S_{3,2,1 / 6}}^{2}\right)\left(k^{2}-m_{d_{i}}^{2}\right)\left(k^{2}-m_{S_{6,3,1 / 3}}^{2}\right)\left((q+k)^{2}-m_{d_{j}}^{2}\right)} .
\end{aligned}
$$

Due to the strong hierarchy in down-type quark masses, the integral in eq. (3.3) is completely dominated by the contributions from bottom quarks, unless the couplings $Y_{Q Q S}^{i j}$, $Y_{\psi d S}^{k i}$ and $Y_{L d S}^{\beta j}$ follow an equally strong inverse hierarchy. We have thus taken into account only the contributions from bottom quark exchange in our numerical evaluation. Since it is convenient to rewrite eq. (3.3) in terms of dimensionless parameters, we define $z, r$ and $t_{k}$ as

$$
z \equiv \frac{m_{S_{3,2,1 / 6}}^{2}}{m_{b}^{2}}, \quad r \equiv \frac{m_{S_{6,3,1 / 3}}^{2}}{m_{b}^{2}}, \quad \text { and } \quad t_{k} \equiv \frac{m_{\psi_{k}}^{2}}{m_{b}^{2}} .
$$

Rescaling the loop momenta, the integral can then be written as:

$$
\hat{I}\left(t_{k}, z, 1, r\right)=\frac{1}{\pi^{4}} \int d^{4} q \int d^{4} k \frac{1}{\left(q^{2}-t_{k}\right)\left(q^{2}-z\right)\left(k^{2}-1\right)\left(k^{2}-r\right)\left((q+k)^{2}-1\right)} .
$$

This integral has been analytically calculated several times in literature. We follow the procedure outlined in [62], based on the calculations of [82]. We will fit the neutrino mass calculated with eq. (3.2) to neutrino oscillation data. The discussion depends on the number of copies of the fermion mediator $\psi_{6,2,-1 / 6}$; as mentioned above we will discuss two different scenarios in the following subsections.

The rate of the LFV process $\mu \rightarrow e \gamma$ has also been calculated several times in literature. We adapt the general formulas shown in [84] for our particular case. The amplitude for $\mu \rightarrow e \gamma$ decay is given by

$$
\mathcal{M}(\mu \rightarrow e \gamma)=e \sigma_{R} \epsilon_{\alpha}^{*} q_{\beta} \bar{u}\left(p_{e}\right) i \sigma^{\alpha \beta} u\left(p_{\mu}\right),
$$

where $e$ is the electric charge, $\epsilon_{\alpha}$ is the photon polarization vector, $q_{\beta}$ is the momentum of photon, and $\sigma^{\alpha \beta} \equiv(i / 2)\left[\gamma^{\alpha}, \gamma^{\beta}\right]$. There are two contributions to the coefficient $\sigma_{R}$ in the model we are discussing; one is the one-loop diagram with the diquark and the exotic fermion, the other is that with a bottom quark and the leptoquark. The total $\sigma_{R}$ is given by $\sigma_{R}=i \frac{m_{\mu}}{16 \pi^{2}}\left[18 \sum_{k}\left(Y_{L \psi S}\right)_{\mu k}\left(Y_{L \psi S}^{\dagger}\right)_{k e} \frac{F_{2}\left(x_{k}\right)}{m_{S_{6,3,1 / 3}}^{2}}+\left(Y_{L d S}\right)_{\mu 3}\left(Y_{L d S}^{\dagger}\right)_{3 e} \frac{2 F_{2}\left(x_{S}\right)-F_{1}\left(x_{S}\right)}{m_{S_{3,2,1 / 6}}^{2}}\right]$,

where $x_{k} \equiv \frac{m_{\psi_{k}}^{2}}{m_{S_{6,3,1 / 3}}^{2}}$ and $x_{S} \equiv \frac{m_{b}^{2}}{m_{S_{3,2,1 / 6}}^{2}}$. The functions $F_{1}(x)$ and $F_{2}(x)$ are defined as

$$
\begin{aligned}
& F_{1}(x)=\frac{x^{2}-5 x-2}{12(x-1)^{3}}+\frac{x \ln x}{2(x-1)^{4}}, \\
& F_{2}(x)=\frac{2 x^{2}+5 x-1}{12(x-1)^{3}}-\frac{x^{2} \ln x}{2(x-1)^{4}}
\end{aligned}
$$


which are presented in eqs. (40) and (41) in [84]. The branching ratio for the $\mu \rightarrow e \gamma$ process, neglecting the electron mass, can then be expressed with the coefficient $\sigma_{R}$ as

$$
\operatorname{Br}(\mu \rightarrow e \gamma) \simeq \frac{48 \pi^{3} \alpha\left|\sigma_{R}\right|^{2}}{G_{F}^{2} m_{\mu}^{2}}
$$

where $\alpha$ is the fine-structure constant.

\subsection{One generation of $\psi_{6,2,1 / 6}$}

The analysis presented in this section uses very similar methods to the one in ref. [16], where double beta decay and LFV is discussed in a 1-loop neutrino mass model containing colour octets. We will first consider a variant of the model, in which there is only one copy of the fermion mediator $\psi_{6,2,1 / 6}$. The expression for the neutrino mass matrix in this case is given by suppressing the index for $\psi$ in eq. (3.2), which gives

$$
\left(m_{\nu}\right)_{\alpha \beta}=\left[\left(Y_{L \psi S}\right)_{\alpha}\left(Y_{L d S}\right)_{\beta 3}+\left(Y_{L \psi S}\right)_{\beta}\left(Y_{L d S}\right)_{\alpha 3}\right] \mathcal{F}
$$

where

$$
\mathcal{F}=\frac{N_{c} m_{\psi}}{\left(16 \pi^{2}\right)^{2}}\left(Y_{Q Q S}\right)_{33}\left(Y_{\psi d S}\right)_{3} \hat{I}(t, z, 1, r) .
$$

Since $\operatorname{det}\left(m_{\nu}\right)=0$ in this case, this version of the model can fit only to the hierarchical neutrino mass spectra (both of the normal and the inverse type), but not to the degenerate spectrum. ${ }^{11}$ The eigenvalues of eq. (3.11) can be easily found to be:

$$
m_{\nu_{1(3)}}=0, \quad m_{\nu_{2,3(1,2)}}=\left[\sum_{\alpha}\left(Y_{L \psi S}\right)_{\alpha}\left(Y_{L d S}\right)_{\alpha 3} \mp \sqrt{\sum_{\alpha}\left|\left(Y_{L \psi S}\right)_{\alpha}\right|^{2} \sum_{\alpha}\left|\left(Y_{L d S}\right)_{\alpha 3}\right|^{2}}\right] \mathcal{F}
$$

for normal hierarchy (inverted hierarchy). In figure 13, we give typical values for the common factor $\mathcal{F}$, which are calculated with the assumption of a nearly degenerate spectrum of heavy particles with the mass scale $M_{\text {eff }} \equiv m_{\psi} \simeq m_{S_{3,2,1 / 6}} \simeq m_{S_{6,3,1 / 3}}$ and $\left(Y_{Q Q S}\right)_{33}=\left(Y_{\psi d S}\right)_{3}=1$. From eq. (3.13) and figure 13, one can estimate the constraints from neutrino masses on the size of the Yukawa couplings. In order to reproduce the neutrino mass suggested by atmospheric neutrino oscillation $\left(m_{\nu_{3}} \sim 0.05 \mathrm{eV}\right)$, keeping the common mass scale $M_{\text {eff }}$ at $1 \mathrm{TeV}$, the Yukawa couplings $Y_{L \psi S}^{\alpha}$ and $Y_{L d S}^{\beta}$ must be set typically to $\mathcal{O}\left(10^{-2}\right)$. Although the eigenvectors of eq. (3.11) can be calculated analytically, numerical exercises might be more helpful to grasp phenomenological aspects of the model. In the following, we will generate random sets of Yukawa couplings $\left(Y_{L \psi S}\right)_{\alpha}$ and $\left(Y_{L d S}\right)_{\beta 3}$ under the condition that they reproduce the latest neutrino oscillation data [83] within 3 $\sigma$ C.L. We will only show plots with the Yukawa couplings that fit the normal hierarchical neutrino spectrum, because plots for the inverse hierarchical case look qualitatively similar.

Let us start the discussion with double beta decay. The half-life of $0 \nu \beta \beta$ induced by the Majorana mass of neutrino is proportional to the inverse-square of the effective

\footnotetext{
${ }^{11}$ However, we remind that this is true, only when contributions to neutrino masses from the first and the second generation quarks are negligible.
} 


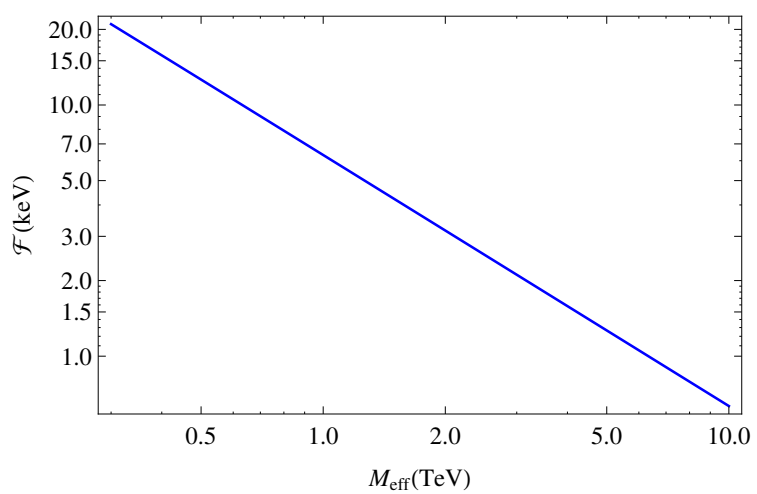

Figure 13. The prefactor $\mathcal{F}$, defined in eq. (3.12), for $\left(Y_{Q Q S}\right)_{33}=\left(Y_{\psi d S}\right)_{3}=1$ in units of $\mathrm{keV}$ as a function of $M_{\text {eff }}$ in TeV. Here, $M_{\text {eff }}=m_{\psi} \simeq m_{S_{3,2,1 / 6}} \simeq m_{S_{6,3,1 / 3}}$.

neutrino mass:

$$
T_{1 / 2}^{0 \nu \beta \beta} \propto\left[\left(m_{\nu}\right)_{e e}\right]^{-2}
$$

For the normal hierarchy case, the effective mass is roughly given as $\left(m_{\nu}\right)_{e e} \sim s_{12}^{2} \sqrt{\Delta m_{21}^{2}} \sim$ $3 \times 10^{-3} \mathrm{eV}$, which results in half-lives of the order of $T_{1 / 2}^{0 \nu \beta \beta} \sim 10^{29}$ ys. For the inverse hierarchy case, one finds $\left(m_{\nu}\right)_{e e} \sim \sqrt{\Delta m_{31}^{2}} \sim 5 \times 10^{-2} \mathrm{eV}$, which leads to $T_{1 / 2}^{0 \nu \beta \beta} \sim 10^{27}$ ys. The current experimental limits to the half-life of ${ }^{136} \mathrm{Xe}$ and ${ }^{76} \mathrm{Ge}$ are of the order of $T_{1 / 2}^{0 \nu \beta \beta} \sim(1-2) \times 10^{25}$ ys $[2,3,85]$, while the next round of experiments could reach eventually $T_{1 / 2}^{0 \nu \beta \beta} \sim 10^{27}$ ys. Therefore, only the inverse hierarchical case can result in measurable half-lifes.

The short range-contribution due to the $d=9$ operator (cf. eq. (2.20)) is proportional to the following combinations of the parameters:

$$
T_{1 / 2}^{0 \nu \beta \beta} \propto\left[\frac{\left(Y_{Q Q S}\right)_{11}\left(Y_{L \psi S}\right)_{e}\left(Y_{\psi d S}\right)_{1}\left(Y_{L d S}\right)_{e 1}}{M_{\mathrm{eff}}^{5}}\right]^{-2},
$$

i.e., while the neutrino mass matrix is dominated by Yukawa couplings of the third quark generation, double beta decay is sensitive only to the Yukawa couplings that couple to the first generation quarks. To discuss the relation between these two contributions to $0 \nu \beta \beta$, we introduce a scaling factor

$$
\eta_{31} \equiv\left[\frac{\left(Y_{Q Q S}\right)_{11}\left(Y_{\psi d S}\right)_{1}\left(Y_{L d S}\right)_{e 1}}{\left(Y_{Q Q S}\right)_{33}\left(Y_{\psi d S}\right)_{3}\left(Y_{L d S}\right)_{e 3}}\right]^{1 / 3},
$$

i.e., $\eta_{31}=1$ corresponds to quark flavour universality in the Yukawa couplings. In figure 14, we calculate half-lives induced from the short-range contribution with randomly generated Yukawa couplings, assuming different values of $\eta_{31} \in\{1,5,10,50\}$. Taking $\eta_{31}=1$, we find quite long half-lives, too large to be measured in realistic experiments. On the other hand, with $\eta_{31}=10$, we find a lower limit on $M_{\text {eff }}$, which is approximately $M_{\text {eff }} \gtrsim 400 \mathrm{GeV}$. This is still not competitive with leptoquark searches at the LHC, which places constraints on the masses of leptoquarks at $m_{S_{3,2,1 / 6}} \sim(600-1000) \mathrm{GeV}$ (depending on generation) 


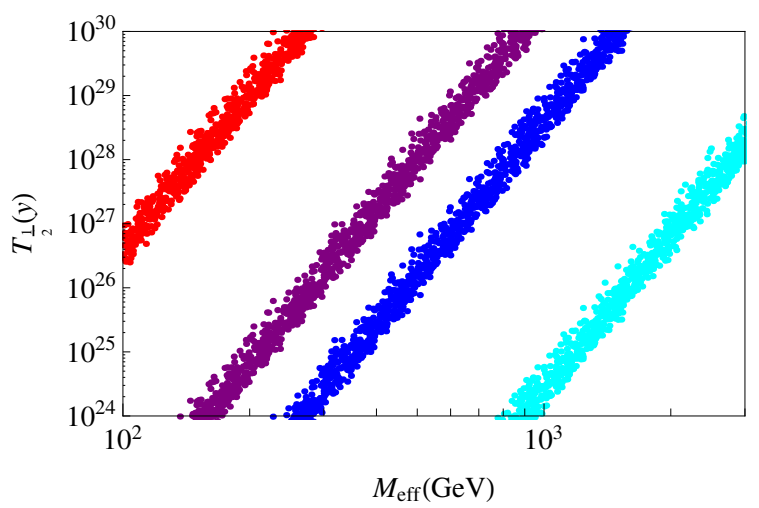

Figure 14. Calculated half-lives for $0 \nu \beta \beta$ decay of ${ }^{136} \mathrm{Xe}$, considering only the short range contribution to the decay rate. The different colours correspond to (from left to right) $\eta_{31}=1,5,10$ and 50. If the third and first generation couplings are of the same order, $0 \nu \beta \beta$ decay will have an immeasurably large half-life in the variant of the model with only one copy of $\psi_{6,2,1 / 6}$.

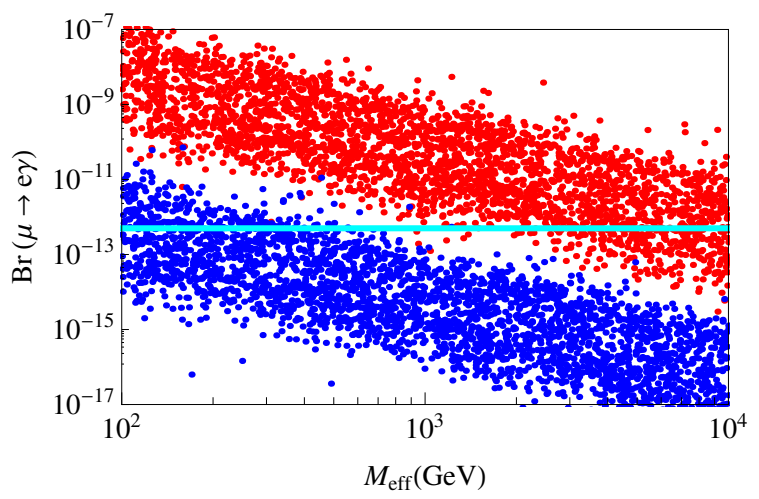

Figure 15. Branching ratio $\operatorname{Br}(\mu \rightarrow e \gamma)$ as a function of $M_{\text {eff }}$ in $\mathrm{GeV}$. Red (blue) points have been calculated with $\left(Y_{Q Q S}\right)_{33}=\left(Y_{\psi d S}\right)_{3}=10^{-2}\left(10^{-1}\right)$. The horizontal line is the experimental upper limit from the MEG experiment [89].

already in the first run [86-88] Thus, it is reasonable to conclude that, as for the mass mechanism contribution, the short-range contribution to the half-life is also expected to be too long to be measured in the near future in this variant of the model, unless $\eta_{31}$ is very large (i.e., for highly inverse hierarchical Yukawa couplings in terms of the quark generations).

Finally, we discuss briefly the LFV process $\mu \rightarrow e \gamma$. While the neutrino mass matrix is proportional to the combination of the Yukawa couplings $\left(Y_{L \psi S}\right)_{\alpha}\left(Y_{L d S}\right)_{\beta 3}\left(Y_{Q Q S}\right)_{33}\left(Y_{\psi d S}\right)_{3}$, the branching ratio of $\operatorname{Br}(\mu \rightarrow e \gamma)$ depends only on $\left|\left(Y_{L \psi S}\right)_{2}\left(Y_{L \psi S}^{\dagger}\right)_{1}\right|^{2}$ and $\left|\left(Y_{L d S}\right)_{23}\left(Y_{L d S}^{\dagger}\right)_{13}\right|^{2}$. In figure 15, we show $\operatorname{Br}(\mu \rightarrow e \gamma)$ for two different choices of the set of $\left(Y_{Q Q S}\right)_{33}$ and $\left(Y_{\psi d S}\right)_{3}$, as a function of $M_{\text {eff }}$, assuming again that the mass spectra of heavy particles are nearly degenerate for simplicity. With the choice $\left(Y_{Q Q S}\right)_{33}=\left(Y_{\psi d S}\right)_{3}=10^{-2}$ the LFV process can place a bound on $M_{\text {eff }}$ of roughly $M_{\text {eff }} \gtrsim \mathrm{TeV}$. However, the bound depends strongly on the exact choice of the remaining Yukawa couplings $Y_{L \psi S}$ and $Y_{L d S}$. On the other hand, the LFV process can exclude only 
few parameter points in the case of $\left(Y_{Q Q S}\right)_{33}=\left(Y_{\psi d S}\right)_{3}=10^{-1}$, and no useful limit on $M_{\text {eff }}$ can be derived.

As we have seen in this subsection, this variant of the model can reproduce oscillation data without running into conflict with LFV searches. However, it is interesting to note that the $\left(Y_{Q Q S}\right)_{33}$ interaction with the size required for reproducing neutrino masses will result in sizeable decay rates of the diquark into third generation quarks (both tops and bottoms), which should be testable at the LHC.

\subsection{Three generations of $\psi_{6,2,1 / 6}$}

Next, we examine the model with more than one copy of the fermion mediator $\psi_{6,2,-1 / 6}$, which can fit not only hierarchical neutrino mass spectra, but also a quasi-degenerate spectrum. Here, we introduce three copies of $\psi_{6,2,-1 / 6}$, motivated by the observed generations of SM fermions.

To simplify the following discussion, we adopt the following ansatz in the flavour structure of the Yukawa couplings: ${ }^{12}$

$$
\left(Y_{L d S}\right)_{\alpha 3}\left(Y_{\psi d S}\right)_{k 3}=y\left(Y_{L \psi S}\right)_{\alpha k}
$$

With this ansatz, all the flavour structure relevant to phenomenology can be represented with only one vector (apart from a possible normalization factor $y$ ). The neutrino mass matrix can then be cast into the form:

$$
\left(m_{\nu}\right)_{\alpha \beta}=(\Lambda)_{\alpha k} \hat{I}_{k}\left(\Lambda^{T}\right)_{k \beta},
$$

where the $\Lambda$ is defined as

$$
\Lambda_{\alpha k} \equiv\left(Y_{L \psi S}\right)_{\alpha k}=\frac{1}{y}\left(Y_{L d S}\right)_{\alpha 3}\left(Y_{\psi d S}\right)_{k 3}
$$

and $\hat{I}$ is given as

$$
\hat{I}_{k}=\frac{2 N_{c} m_{\psi_{k}}}{\left(16 \pi^{2}\right)^{2}} y\left(Y_{Q Q S}\right)_{33} I\left(z, 1, t_{k}, r\right)
$$

Comparing eq. (3.18) with the neutrino mass and mixing matrix, we can find the direct relation between $\Lambda$ and the measured neutrino data. Following the procedure originally developed by Casas and Ibarra for seesaw type-I [90], we parametrize $\Lambda$ as

$$
\left(\Lambda^{T}\right)_{k \alpha}=\left(\sqrt{\hat{I}^{-1}}\right)_{k} R_{k i}\left(\sqrt{\hat{m}_{\nu}}\right)_{i}\left(U_{\nu}^{\dagger}\right)_{i \alpha} .
$$

Here, $\hat{m}_{\nu}$ is the matrix of eigenvalues of $m_{\nu}$, which is diagonalized with the neutrino mixing matrix $U_{\nu}$ via

$$
\left(U_{\nu}^{T}\right)_{i \alpha}\left(m_{\nu}\right)_{\alpha \beta}\left(U_{\nu}\right)_{\beta j} \equiv \hat{m}_{\nu}=\operatorname{diag}\left(m_{\nu_{1}} m_{\nu_{2}} m_{\nu_{3}}\right)
$$

\footnotetext{
${ }^{12}$ This ansatz can be justified by introducing a flavour symmetry with flavour-charged scalar (flavon) fields.
} 
for which we use the following standard parametrization

$$
U_{\nu}=\left(\begin{array}{ccc}
c_{12} c_{13} & s_{12} c_{13} & s_{13} e^{i \delta} \\
-s_{12} c_{23}-c_{12} s_{23} s_{13} e^{-i \delta} & c_{12} c_{23}-s_{12} s_{23} s_{13} e^{-i \delta} & s_{23} c_{13} \\
s_{12} s_{23}-c_{12} c_{23} s_{13} e^{-i \delta} & -c_{12} s_{23}-s_{12} c_{23} s_{13} e^{-i \delta} & c_{23} c_{13}
\end{array}\right)\left(\begin{array}{ccc}
e^{i \alpha_{1}} & 0 & 0 \\
0 & e^{i \alpha_{2}} & 0 \\
0 & 0 & 1
\end{array}\right)
$$

$c_{i j}=\cos \theta_{i j}, s_{i j}=\sin \theta_{i j}$ with the mixing angles $\theta_{i j}, \delta$ is the Dirac phase and $\alpha_{1}, \alpha_{2}$ are Majorana phases. Finally, $R$ is a complex orthogonal matrix which satisfies the condition $R^{T} R=1$. We use the following parametrization for the $R$ matrix in terms of three complex angles $\theta_{1}, \theta_{2}$, and $\theta_{3}$ as

$$
R=\left(\begin{array}{ccc}
c_{2} c_{3}-c_{1} s_{3}-s_{1} s_{2} c_{3} & s_{1} s_{3}-c_{1} s_{2} c_{3} \\
c_{2} s_{3} & c_{1} c_{3}-s_{1} s_{2} s_{3} & -s_{1} c_{3}-c_{1} s_{2} s_{3} \\
s_{2} & s_{1} c_{2} & c_{1} c_{2}
\end{array}\right)
$$

After fitting the neutrino oscillation data with the parametrization shown above, there remain $y,\left(Y_{Q Q S}\right)_{33}$ and the masses $m_{S_{6,3,1 / 3}}, m_{S_{3,2,1 / 6}}, m_{\psi_{k}}$ as free parameters. For the calculation of the short-range contribution to the $0 \nu \beta \beta$ decay, we also have the parameter $\eta_{31}$. For simplicity, we set $y=1$ and assume again a nearly degenerate spectrum for heavy particles, which is parameterized with $M_{\text {eff. }}$ We can then calculate half-lives $T_{1 / 2}^{0 \nu \beta \beta}$ for both neutrino mass mechanism and the short-range contribution, as a function of $m_{\nu_{1}}$, $M_{\text {eff }}$ and $\eta_{31}$. In figure 16, we fixed the oscillation parameters $s_{13}^{2}, \Delta m_{31}^{2}$ and $\Delta m_{21}^{2}$ at their best-fit values, while $s_{23}^{2}=1 / 2$ and $s_{12}^{2}=1 / 3$ and set $\delta$ as well as the Majorana phases to zero, just to sketch out some phenomenological aspects of this example. Each panel shows the half-life of the short-range contribution for $0 \nu \beta \beta$ decay of ${ }^{136} \mathrm{Xe}$ as a function of $\eta_{31}$ (top panel), $M_{\text {eff }}$ (middle panel) and $m_{\nu_{1}}$ (bottom panel). In each panel, we examine several choices for the remaining parameters, which are explained in the figure caption. The corresponding half-lives induced from the mass mechanism are also indicated. As shown in figure 16, half-lives can vary over many orders of magnitude with the choice of parameters. The amplitudes induced from the mass mechanism becomes the same order as that from the short-range, when $\eta_{31} \sim 2.7(6.5)$ for $M_{\mathrm{eff}}=0.5 \mathrm{TeV}(1 \mathrm{TeV})$. As in the case with only one generation of $\psi_{6,2,1 / 6}$, the mass mechanism dominates the $0 \nu \beta \beta$, if the ratio $\eta_{31}$ is taken to be unity and the heavy mass scale $M_{\text {eff }}$ is given at the typical LHC search sensitivities. However, since the three-generation case can fit the quasi-degenerate neutrino spectrum, $0 \nu \beta \beta$ decay half-lives can be much shorter than in the one generation case and can saturate the experimental bound.

We now turn to $\operatorname{Br}(\mu \rightarrow e \gamma)$. Again, as in the one generation case, the neutrino mass matrix depends on Yukawa couplings, but is not directly related to $\operatorname{Br}(\mu \rightarrow e \gamma)$. Therefore, we have always the freedom to adjust $\left(Y_{\psi d S}\right)_{k 3}$ and $\left(Y_{Q Q S}\right)_{33}$ so as to fit the neutrino masses. The other Yukawa couplings are then fixed by the neutrino data (and the choice of $M_{\text {eff }}$ ), and we can use them to calculate $\operatorname{Br}(\mu \rightarrow e \gamma)$. Figure 17 shows some examples with a value of $M_{\text {eff }}=1 \mathrm{TeV}$. The plots show that constraints from $\operatorname{Br}(\mu \rightarrow e \gamma)$ can be easily fulfilled. For this choice of $M_{\text {eff }}$, only if both $\left(Y_{Q Q S}\right)_{33}$ and $\left(Y_{\psi d S}\right)_{13}$ are set to order $\mathcal{O}\left(10^{-2}\right)$ or lower, the predicted $\operatorname{Br}(\mu \rightarrow e \gamma)$ can saturate the experimental bound. 

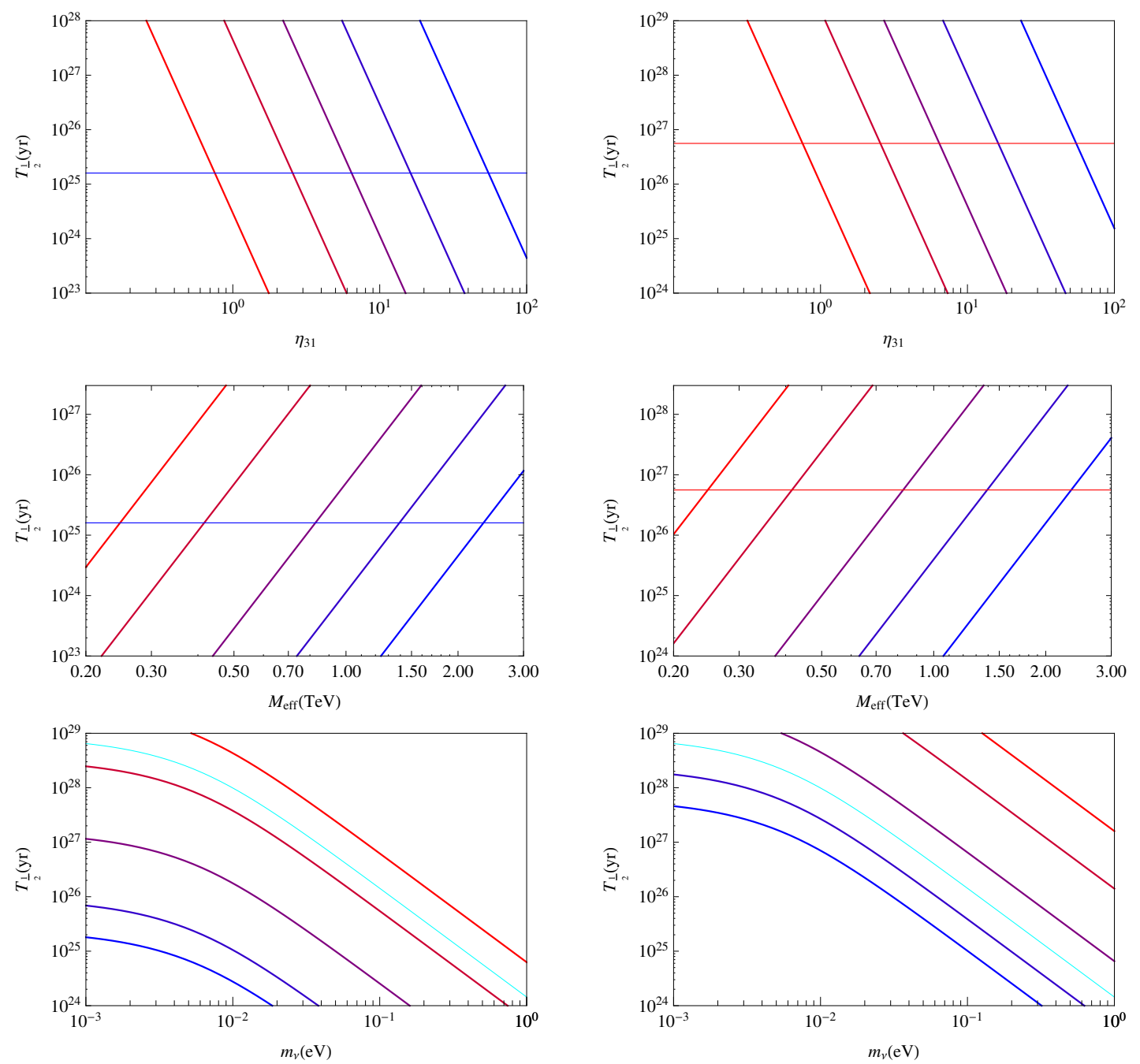

Figure 16. Calculated half-lives for $0 \nu \beta \beta$ decay of ${ }^{136} \mathrm{Xe}$, considering only the short-range contribution to the decay rate. The various plots show from top to bottom: $T_{1 / 2}$ versus $\eta_{31}, M_{\text {eff }}$ and $m_{\nu_{1}}$, for a fixed set of neutrino oscillation parameters and different choices of $\eta_{31}, M_{\text {eff }}$ and $m_{\nu_{1}}$ as follows: in the top plots to the left (right) $m_{\nu_{1}}=0.3 \mathrm{eV}(0.05 \mathrm{eV})$, different lines show different choices of $M_{\text {eff }}$; from left to right: $M_{\text {eff }}=0.2,0.5,1,2$ and $5 \mathrm{TeV}$. In the middle panel, to the left (right) $m_{\nu_{1}}=0.3 \mathrm{eV}(0.05 \mathrm{eV})$, different lines show different choices of $\eta_{31}$; from left to right: $\eta_{31}=1,2,5,10$ and 20 . In the lower panel, to the left (right): $M_{\text {eff }}=0.5 \mathrm{TeV}(1 \mathrm{TeV})$, different lines are for different choices for $\eta_{31}$; from top to bottom: $\eta_{31}=2,3,5,8$ and 10 . For comparison we also show the half-lives for the neutrino mass mechanism as horizontal lines in the top and middle panel and as cyan lines in the lower panel. Oscillation parameters are $s_{13}^{2}, \Delta m_{31}^{2}$ and $\Delta m_{21}^{2}$ at their best-fit values, while $s_{23}^{2}=1 / 2$ and $s_{12}^{2}=1 / 3$ for the case of normal hierarchy.

\section{Conclusions and discussion}

We have discussed the relation between the $d=9$ short-range contributions to the $0 \nu \beta \beta$ decay amplitude with neutrino mass models. All contributions to $0 \nu \beta \beta$ decay violate lepton number and, therefore, generate also Majorana neutrino masses. We have classified 

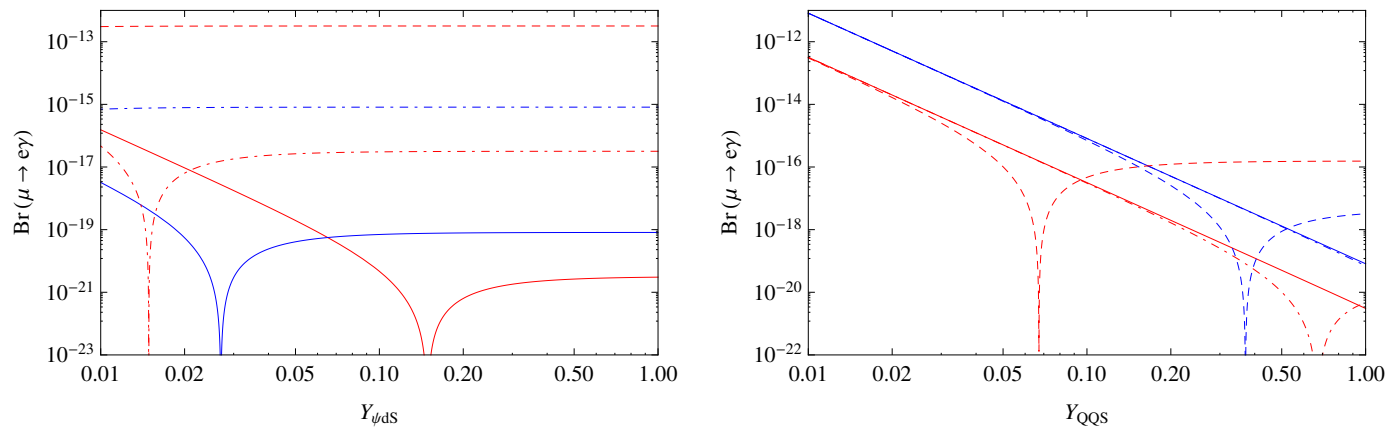

Figure 17. $\operatorname{Br}(\mu \rightarrow e \gamma)$ versus $Y_{\psi d S} \equiv\left(Y_{\psi d S}\right)_{13}$ (left) and $Y_{Q Q S} \equiv\left(Y_{Q Q S}\right)_{33}$ (right), for a fixed choice of $M_{\text {eff }}=1 \mathrm{TeV}$, neutrino oscillation parameters as in figure 16 and $m_{\nu_{1}}=0.05 \mathrm{eV}$ (red lines) and $m_{\nu_{1}}=0.3 \mathrm{eV}$ (blue lines). Full, dot-dashed and dashed lines are for $Y_{\psi d S}$ (right) and $Y_{Q Q S}$ (left) equal to $1,10^{-1}$ and $10^{-2}$ respectively. $\operatorname{Br}(\mu \rightarrow e \gamma)$ can saturate the experimental bound only for small values of these couplings, since smaller values of $Y_{\psi d S}$ and $Y_{Q Q S}$ require larger values for $Y_{L \psi S}$ and $Y_{L d S}$, in order to fit neutrino data.

all possible (scalar-mediated) short-range contributions to the decay rate according to the loop level, at which the corresponding models will generate Majorana neutrino masses. Possibilities range from tree-level to 4-loop neutrino masses. For each case we have discussed one example briefly and given estimates of the typical constraints imposed by both the short-range contribution and the mass mechanism. Generally, one expects that for models with tree- or 1-loop neutrino masses, the short-range $0 \nu \beta \beta$ decay amplitude will be sub-dominant to the mass mechanism. For 2-loop models short-range $0 \nu \beta \beta$ decay amplitude and mass mechanism can be comparable, while for 3-loop and 4-loop models the short-range part of the amplitude will dominate.

We have also discussed one particular example of a 2-loop model in more detail. Here, we have shown different parts of parameter space where mass mechanism or short-range amplitude dominant can each be dominant. In the study, we have taken recent neutrino oscillation data and constraints from LFV experiments into consideration.

In the appendix we give the full list of decompositions, classified according to our scheme, in tabular form.

\section{Acknowledgments}

T.O. is grateful to Prof. Junji Hisano for insightful comments on flavour structure of the effective interactions. J.C.H. thanks the IFIC for hospitality during his stay. Work supported by the Spanish grants FPA2014-58183-P and Multidark CSD2009-00064 (MINECO), and PROMETEOII/2014/084 (Generalitat Valenciana), by Fondecyt (Chile) under grants 11121557 and by CONICYT (Chile) project 791100017. The research of T.O. is supported by JSPS Grants-in-Aid for Scientific Research on Innovative Areas Unification and Development of the Neutrino Science Frontier Number 2610 5503. The research of F.A.P.S. is supported by the Brazilian Research Council CNPq. 


\section{A Summary tables}

Here, we give tables in which all possible scalar short-range decompositions are classified according to the loop level at which they will generate neutrino masses. The decompositions which generate neutrino masses at tree, 1-loop, 2-loop, 3-loop and 4-loop level are listed in tables 1 to 7 . The identification number given to each decomposition is defined in [31]. The notation T-I and T-II refers to the two possible topologies of the decompositions of $d=90 \nu \beta \beta$ effective operators, and the BL number is the Babu-Leung classification of the effective neutrino mass operator, given in [35]. The columns "Add. Int." specify additional interactions, with respect to those appearing in the decomposition. While they do not appear directly in the $0 \nu \beta \beta$ diagram, these additional interaction can not be forbidden by any symmetry, without forbidding the corresponding $0 \nu \beta \beta$ decay decomposition at the same time. Once present, they generate a neutrino mass diagrams at the quoted loop level. The columns "Diagram" specify the topology of the neutrino mass diagram, for example "type-I" for seesaw type-I and so forth. The identification numbers for the 1-loop and 2loop neutrino mass diagrams are taken from the general topology classification given in [77] and [62], respectively.

Here, we briefly comment on "associated operators". As discussed in ref. [77], some of the decompositions generate not only the original effective operator but also necessarily generate other operators, when all possible contractions are carried out. We call this associated operators. For example, the decomposition T-I-2-iii-a of the BL \#19 operator consists of the following fundamental interactions,

$$
\begin{aligned}
\mathcal{L}_{\text {T-I-2-iii-a }}= & Y_{L d S}\left(\bar{L} d_{R}\right) \cdot S_{\overline{3}, 2,-1 / 6}+Y_{Q \psi S}(\vec{Q} \vec{\lambda} \vec{\psi}) \cdot S_{\overline{3}, 2,-1 / 6}^{\dagger} \\
& +Y_{\psi d S} S_{\overline{3}, 1,1 / 3}\left(\overrightarrow{\bar{\psi}} \vec{\lambda} d_{R}\right)+Y_{u e S}\left(\overline{u_{R}} e_{R}^{c}\right) S_{\overline{3}, 1,1 / 3}^{\dagger}+\text { H.c. },
\end{aligned}
$$

where $\vec{\lambda}$ is the Gell-Mann matrices. The first two interactions, together with the Majorana mass of the fermion $\psi_{8,1,0}$ result in the BL \#11 operator $\left(\bar{L} d_{R}\right)(\bar{Q})(\bar{Q})\left(\bar{L} d_{R}\right)$. In the same way, the last two interactions lead to the $d=9$ lepton number violating effective operator $\left(\overline{u_{R} e_{R}}\right)\left(d_{R}\right)\left(d_{R}\right)\left(\overline{u_{R} e_{R}}\right)$, which is $\mathcal{O}_{-}$in eq. (2.4). All the decompositions accompanied by associated operators were listed in tables of ref. [77]. We take into account the associated effective operators in our classification scheme. In short, if the associated operator generates neutrino masses at a lower loop level than the original one, we classify the decomposition with the loop level of the associated operator. The Lagrangian for a concrete example is given in eq. (A.1). Here, although the original effective operator BL \#19 gives neutrino masses only at the 3-loop level, the decomposition T-I-2-iii-b of BL \#19 also produces BL \#11, and it generates the 2-loop neutrino mass diagram with the help of the SM Yukawa interactions. Therefore, we list the decomposition T-I-2-iii-b of BL \#19 as a 2-loop neutrino mass model in table 4. More examples are given in the tables. 


\begin{tabular}{|c|c|c|c|c|c|c|c|}
\hline T-I \# & Op. & BL \# & $S$ & $\psi$ & $S^{\prime}$ & Diagram & Add. Int. \\
\hline $1-\mathrm{i}$ & $(\bar{u} d)(\bar{e})(\bar{e})(\bar{u} d)$ & $11,12,14$ & $(1,2)_{+1 / 2}$ & $(1,1)_{0}$ & $(1,2)_{-1 / 2}$ & type I & $\bar{L} \psi_{110} H^{\dagger}$ \\
\hline $1-\mathrm{i}$ & $(\bar{u} d)(\bar{e})(\bar{e})(\bar{u} d)$ & $11,12,14$ & $(1,2)_{+1 / 2}$ & $(1,3)_{0}$ & $(1,2)_{-1 / 2}$ & type III & $\bar{L} \psi_{130} H^{\dagger}$ \\
\hline 1-ii-a & $(\bar{u} d)(\bar{u})(d)(\bar{e} \bar{e})$ & 11,14 & $(1,2)_{+1 / 2}$ & $(3,3)_{+2 / 3}$ & $(1,3)_{+1}$ & type II & $S_{131} H^{\dagger} H^{\dagger}$ \\
\hline $1-\mathrm{ii}-\mathrm{a}$ & $(\bar{u} d)(\bar{u})(d)(\bar{e} \bar{e})$ & 11,14 & $(8,2)_{+1 / 2}$ & $(3,3)_{+2 / 3}$ & $(1,3)_{+1}$ & type II & $S_{131} H^{\dagger} H^{\dagger}$ \\
\hline $1-\mathrm{ii}-\mathrm{a}$ & $(\bar{u} d)(\bar{u})(d)(\bar{e} \bar{e})$ & 12,14 & $(1,2)_{+1 / 2}$ & $(3,2)_{+7 / 6}$ & $(1,3)_{+1}$ & type II & $S_{131} H^{\dagger} H^{\dagger}$ \\
\hline $1-\mathrm{ii}-\mathrm{a}$ & $(\bar{u} d)(\bar{u})(d)(\bar{e} \bar{e})$ & 12,14 & $(8,2)_{+1 / 2}$ & $(3,2)_{+7 / 6}$ & $(1,3)_{+1}$ & type II & $S_{131} H^{\dagger} H^{\dagger}$ \\
\hline $1-\mathrm{ii}-\mathrm{b}$ & $(\bar{u} d)(d)(\bar{u})(\bar{e} \bar{e})$ & 12,14 & $(1,2)_{+1 / 2}$ & $(\overline{3}, 3)_{+1 / 3}$ & $(1,3)+1$ & type II & $S_{131} H^{\dagger} H^{\dagger}$ \\
\hline $1-i i-b$ & $(\bar{u} d)(d)(\bar{u})(\bar{e} \bar{e})$ & 12,14 & $(8,2)_{+1 / 2}$ & $(\overline{3}, 3)_{+1 / 3}$ & $(1,3)_{+1}$ & type II & $S_{131} H^{\dagger} H^{\dagger}$ \\
\hline $1-i i-b$ & $(\bar{u} d)(d)(\bar{u})(\bar{e} \bar{e})$ & 11,14 & $(1,2)_{+1 / 2}$ & $(\overline{3}, 2)_{+5 / 3}$ & $(1,3)_{+1}$ & type II & $S_{131} H^{\dagger} H^{\dagger}$ \\
\hline $1-i i-b$ & $(\bar{u} d)(d)(\bar{u})(\bar{e} \bar{e})$ & 11,14 & $(8,2)_{+1 / 2}$ & $(\overline{3}, 2)_{+5 / 3}$ & $(1,3)_{+1}$ & type II & $S_{131} H^{\dagger} H^{\dagger}$ \\
\hline $2-\mathrm{i}-\mathrm{b}$ & $(\bar{u} d)(\bar{e})(d)(\bar{u} \bar{e})$ & $11,19,14,20$ & $(1,2)_{+1 / 2}$ & $(1,1)_{0}$ & $(\overline{3}, 1)_{+1 / 3}$ & type I & $\bar{L} \psi_{110} H^{\dagger}$ \\
\hline $2-\mathrm{i}-\mathrm{b}$ & $(\bar{u} d)(\bar{e})(d)(\bar{u} \bar{e})$ & 11,14 & $(1,2)_{+1 / 2}$ & $(1,3)_{0}$ & $(\overline{3}, 3)_{+1 / 3}$ & type III & $\bar{L} \psi_{130} H^{\dagger}$ \\
\hline $2-\mathrm{ii}-\mathrm{b}$ & $(\bar{u} d)(\bar{e})(\bar{u})(d \bar{e})$ & 11,14 & $(1,2)_{+1 / 2}$ & $(1,1)_{0}$ & $(3,2)_{+1 / 6}$ & type I & $\bar{L} \psi_{110} H^{\dagger}$ \\
\hline 2 -ii-b & $(\bar{u} d)(\bar{e})(\bar{u})(d \bar{e})$ & 11,14 & $(1,2)_{+1 / 2}$ & $(1,3)_{0}$ & $(3,2)_{+1 / 6}$ & type III & $\bar{L} \psi_{130} H^{\dagger}$ \\
\hline 2-iii-a & $(d \bar{e})(\bar{u})(d)(\bar{u} \bar{e})$ & 11,19 & $(\overline{3}, 2)_{-1 / 6}$ & $(1,1)_{0}$ & $(\overline{3}, 1)_{+1 / 3}$ & type I & $\bar{L} \psi_{110} H^{\dagger}$ \\
\hline 2-iii-a & $(d \bar{e})(\bar{u})(d)(\bar{u} \bar{e})$ & 11 & $(\overline{3}, 2)_{-1 / 6}$ & $(1,3)_{0}$ & $(\overline{3}, 3)_{+1 / 3}$ & type III & $\bar{L} \psi_{130} H^{\dagger}$ \\
\hline 3-ii & $(\bar{u} \bar{u})(d)(d)(\bar{e} \bar{e})$ & 11 & $(6,3)_{+1 / 3}$ & $(3,3)_{+2 / 3}$ & $(1,3)_{+1}$ & type II & $S_{131} H^{\dagger} H^{\dagger}$ \\
\hline 3 -ii & $(\bar{u} \bar{u})(d)(d)(\bar{e} \bar{e})$ & 12 & $(6,1)_{+4 / 3}$ & $(3,2)_{+7 / 6}$ & $(1,3)_{+1}$ & type II & $S_{131} H^{\dagger} H^{\dagger}$ \\
\hline 3-iii & $(d d)(\bar{u})(\bar{u})(\bar{e} \bar{e})$ & 12 & $(\overline{6}, 3)_{-1 / 3}$ & $(\overline{3}, 3)_{+1 / 3}$ & $(1,3)_{+1}$ & type II & $S_{131} H^{\dagger} H^{\dagger}$ \\
\hline 3-iii & $(d d)(\bar{u})(\bar{u})(\bar{e} \bar{e})$ & 11 & $(\overline{6}, 1)_{+2 / 3}$ & $(\overline{3}, 2)_{+5 / 6}$ & $(1,3)_{+1}$ & type II & $S_{131} H^{\dagger} H^{\dagger}$ \\
\hline $4-\mathrm{i}$ & $(d \bar{e})(\bar{u})(\bar{u})(d \bar{e})$ & 11 & $(\overline{3}, 2)_{-1 / 6}$ & $(1,1)_{0}$ & $(3,2)_{+1 / 6}$ & type I & $\bar{L} \psi_{110} H^{\dagger}$ \\
\hline $4-\mathrm{i}$ & $(d \bar{e})(\bar{u})(\bar{u})(d \bar{e})$ & 11 & $(\overline{3}, 2)_{-1 / 6}$ & $(1,3)_{0}$ & $(3,2)_{+1 / 6}$ & type III & $\bar{L} \psi_{130} H^{\dagger}$ \\
\hline $5-\mathrm{i}$ & $(\bar{u} \bar{e})(d)(d)(\bar{u} \bar{e})$ & $11,19,-$ & $(3,1)_{-1 / 3}$ & $(1,1)_{0}$ & $(\overline{3}, 1)_{+1 / 3}$ & type I & $\bar{L} \psi_{110} H^{\dagger}$ \\
\hline $5-\mathrm{i}$ & $(\bar{u} \bar{e})(d)(d)(\bar{u} \bar{e})$ & 11 & $(3,3)_{-1 / 3}$ & $(1,3)_{0}$ & $(\overline{3}, 3)_{+1 / 3}$ & type III & $\bar{L} \psi_{130} H^{\dagger}$ \\
\hline
\end{tabular}

\begin{tabular}{|cccccccc|}
\hline T-II \# & Op. & BL \# & $S$ & $S^{\prime}$ & $S^{\prime \prime}$ & Diagram & Add. Int. \\
\hline 1 & $(\bar{u} d)(\bar{u} d)(\bar{e} \bar{e})$ & $11,12,14$ & $(1,2)_{+1 / 2}$ & $(1,2)_{+1 / 2}$ & $(1,3)_{-1}$ & type II & $S_{13-1} H H$ \\
\hline 1 & $(\bar{u} d)(\bar{u} d)(\bar{e} \bar{e})$ & $11,12,14$ & $(8,2)_{+1 / 2}$ & $(8,2)_{+1 / 2}$ & $(1,3)_{-1}$ & type II & $S_{13-1} H H$ \\
\hline 3 & $(\bar{u} \bar{u})(d d)(\bar{e} \bar{e})$ & 11 & $(6,3)_{+1 / 3}$ & $(\overline{6}, 1)_{+2 / 3}$ & $(1,3)_{-1}$ & type II & $S_{13-1} H H$ \\
\hline 3 & $(\bar{u} \bar{u})(d d)(\bar{e} \bar{e})$ & 12 & $(6,1)_{+4 / 3}$ & $(\overline{6}, 3)_{-1 / 3}$ & $(1,3)_{-1}$ & type II & $S_{13-1} H H$ \\
\hline
\end{tabular}

Table 1. List of the decompositions that generate neutrino masses at tree level. The ID-numbers with "T" are assigned as in ref. [31], and the decomposition is specified in the "Op." column. We also give the ID-numbers of lepton-number-violating effective operators, which are classified as in Babu and Leung [35], in "BL\#". The SM charges of fields appearing in the decomposition are also given. "Diagram" indicates the type of resulting tree-level neutrino mass diagrams: "type I" for type I seesaw mechanism, and so on. In the column "Add. Int.", we give the additional interaction that is missing in the decomposition but is necessary to generate the neutrino mass diagram. For the decompositions in this table, unless some severe fine-tuning of parameters is done, the mass mechanism of double beta decay will dominate over the short-range contributions. 


\begin{tabular}{|c|c|c|c|c|c|c|c|}
\hline T-I \# & Op. & BL \# & $S$ & $\psi$ & $S^{\prime}$ & Diagram & Add. Int. \\
\hline $1-\mathrm{i}$ & $(\bar{u} d)(\bar{e})(\bar{e})(\bar{u} d)$ & $11,12,14$ & $(8,2)_{+1 / 2}$ & $(8,1)_{0}$ & $(8,2)_{-1 / 2}$ & $\mathrm{~T} \nu-3$ & $S_{82 \frac{1}{2}} S_{82 \frac{1}{2}} H^{\dagger} H^{\dagger}$ \\
\hline $1-\mathrm{i}$ & $(\bar{u} d)(\bar{e})(\bar{e})(\bar{u} d)$ & $11,12,14$ & $(8,2)_{+1 / 2}$ & $(8,3)_{0}$ & $(8,2)_{-1 / 2}$ & $\mathrm{~T} \nu-3$ & $S_{82 \frac{1}{2}} S_{82 \frac{1}{2}} H^{\dagger} H^{\dagger}$ \\
\hline $2-\mathrm{i}-\mathrm{a}$ & $(\bar{u} d)(d)(\bar{e})(\bar{u} \bar{e})$ & 11,14 & $(1,2)_{+1 / 2}$ & $(\overline{3}, 2)_{+5 / 6}$ & $(\overline{3}, 1)_{+1 / 3}$ & $\mathrm{~T} \nu$-1-iii & $\bar{d}_{R}^{c} \psi_{\overline{3} 2 \frac{5}{6}} H^{\dagger}$ \\
\hline $2-\mathrm{i}-\mathrm{a}$ & $(\bar{u} d)(d)(\bar{e})(\bar{u} \bar{e})$ & 11,14 & $(1,2)_{+1 / 2}$ & $(\overline{3}, 2)_{+5 / 6}$ & $(\overline{3}, 3)_{+1 / 3}$ & $\mathrm{~T} \nu$-1-iii & $\bar{d}_{R}^{c} \psi_{\overline{3} 2 \frac{5}{6}} H^{\dagger}$ \\
\hline $2-\mathrm{i}-\mathrm{a}$ & $(\bar{u} d)(d)(\bar{e})(\bar{u} \bar{e})$ & 11,14 & $(8,2)_{+1 / 2}$ & $(\overline{3}, 2)_{+5 / 6}$ & $(\overline{3}, 1)_{+1 / 3}$ & $\mathrm{~T} \nu$-1-iii & $\bar{d}_{R}^{c} \psi_{\overline{3} 2 \frac{5}{6}} H^{\dagger}$ \\
\hline $2-\mathrm{i}-\mathrm{a}$ & $(\bar{u} d)(d)(\bar{e})(\bar{u} \bar{e})$ & 11,14 & $(8,2)_{+1 / 2}$ & $(\overline{3}, 2)_{+5 / 6}$ & $(\overline{3}, 3)_{+1 / 3}$ & $\mathrm{~T} \nu$-1-iii & $\bar{d}_{R}^{c} \psi_{\overline{3} 2 \frac{5}{6}} H^{\dagger}$ \\
\hline $2-\mathrm{i}-\mathrm{b}$ & $(\bar{u} d)(\bar{e})(d)(\bar{u} \bar{e})$ & $11,14,19,20$ & $(8,2)_{+1 / 2}$ & $(8,1)_{0}$ & $(\overline{3}, 1)_{+1 / 3}$ & $\mathrm{~T} \nu-3$ & $S_{82 \frac{1}{2}} S_{82 \frac{1}{2}} H^{\dagger} H^{\dagger}$ \\
\hline $2-\mathrm{i}-\mathrm{b}$ & $(\bar{u} d)(\bar{e})(d)(\bar{u} \bar{e})$ & 11,14 & $(8,2)+1 / 2$ & $(8,3)_{0}$ & $(\overline{3}, 3)_{+1 / 3}$ & $\mathrm{~T} \nu-3$ & $S_{82 \frac{1}{2}} S_{82 \frac{1}{2}} H^{\dagger} H^{\dagger}$ \\
\hline 2-ii-a & $(\bar{u} d)(\bar{u})(\bar{e})(d \bar{e})$ & 11,14 & $(1,2)+1 / 2$ & $(3,3)_{+2 / 3}$ & $(3,2)+1 / 6$ & $\mathrm{~T} \nu-1-\mathrm{iii}$ & $\bar{Q} \psi_{\overline{3} 3 \frac{2}{3}} H^{\dagger}$ \\
\hline 2-ii-a & $(\bar{u} d)(\bar{u})(\bar{e})(d \bar{e})$ & 11,14 & $(8,2)_{+1 / 2}$ & $(3,3)_{+2 / 3}$ & $(3,2)_{+1 / 6}$ & $\mathrm{~T} \nu$-1-iii & $\bar{Q} \psi_{\overline{3} 3 \frac{2}{3}} H^{\dagger}$ \\
\hline $2-\mathrm{ii}-\mathrm{b}$ & $(\bar{u} d)(\bar{e})(\bar{u})(d \bar{e})$ & 11,14 & $(8,2)+1 / 2$ & $(8,1)_{0}$ & $(3,2)+1 / 6$ & $\mathrm{~T} \nu-3$ & $S_{82 \frac{1}{2}} S_{82 \frac{1}{2}} H^{\dagger} H^{\dagger}$ \\
\hline $2-\mathrm{ii}-\mathrm{b}$ & $(\bar{u} d)(\bar{e})(\bar{u})(d \bar{e})$ & 11,14 & $(8,2)_{+1 / 2}$ & $(8,3)_{0}$ & $(3,2)_{+1 / 6}$ & $\mathrm{~T} \nu-3$ & $S_{82 \frac{1}{2}} S_{82 \frac{1}{2}} H^{\dagger} H^{\dagger}$ \\
\hline 2-iii-a & $(d \bar{e})(\bar{u})(d)(\bar{u} \bar{e})$ & 11 & $(\overline{3}, 2)_{-1 / 6}$ & $(8,1)_{0}$ & $(\overline{3}, 1)_{+1 / 3}$ & $\mathrm{~T} \nu$-1-ii & $S_{\overline{3} 2-\frac{1}{6}}^{\dagger} S_{\overline{3} 1 \frac{1}{3}} H^{\dagger}$ \\
\hline 2-iii-a & $(d \bar{e})(\bar{u})(d)(\bar{u} \bar{e})$ & 11 & $(\overline{3}, 2)_{-1 / 6}$ & $(8,3)_{0}$ & $(\overline{3}, 3)_{+1 / 3}$ & $\mathrm{~T} \nu$-1-ii & $S_{\overline{3} 2-\frac{1}{6}}^{\dagger} S_{\overline{3} 3 \frac{1}{3}} H^{\dagger}$ \\
\hline 2-iii-a & $(d \bar{e})(\bar{u})(d)(\bar{u} \bar{e})$ & 14 & $(\overline{3}, 2)_{-1 / 6}$ & $(1,2)_{+1 / 2}$ & $(\overline{3}, 1)_{+1 / 3}$ & $\mathrm{~T} \nu$-1-ii & $S_{\overline{3} 2-\frac{1}{6}}^{\dagger} S_{\overline{3} 1 \frac{1}{3}} H^{\dagger}$ \\
\hline 2-iii-a & $(d \bar{e})(\bar{u})(d)(\bar{u} \bar{e})$ & 14 & $(\overline{3}, 2)_{-1 / 6}$ & $(8,2)_{+1 / 2}$ & $(\overline{3}, 1)_{+1 / 3}$ & $\mathrm{~T} \nu$-1-ii & $S_{\overline{3} 2-\frac{1}{6}}^{\dagger} S_{\overline{3} 1 \frac{1}{3}} H^{\dagger}$ \\
\hline 2-iii-a & $(d \bar{e})(\bar{u})(d)(\bar{u} \bar{e})$ & 14 & $(\overline{3}, 2)_{-1 / 6}$ & $(1,2)_{+1 / 2}$ & $(\overline{3}, 3)_{+1 / 3}$ & $\mathrm{~T} \nu$-1-ii & $S_{\overline{3} 2-\frac{1}{6}}^{\dagger} S_{\overline{3} 3 \frac{1}{3}} H^{\dagger}$ \\
\hline 2-iii-a & $(d \bar{e})(\bar{u})(d)(\bar{u} \bar{e})$ & 14 & $(\overline{3}, 2)_{-1 / 6}$ & $(8,2)_{+1 / 2}$ & $(\overline{3}, 3)_{+1 / 3}$ & $\mathrm{~T} \nu$-1-ii & $S_{\overline{3} 2-\frac{1}{6}}^{\dagger} S_{\overline{3} 3 \frac{1}{3}} H^{\dagger}$ \\
\hline 2-iii-b & $(d \bar{e})(d)(\bar{u})(\bar{u} \bar{e})$ & 11 & $(\overline{3}, 2)_{-1 / 6}$ & $(3,2)_{+1 / 6}$ & $(\overline{3}, 1)_{+1 / 3}$ & $\mathrm{~T} \nu$-1-ii & $S_{\overline{3} 2-\frac{1}{6}}^{\dagger} S_{\overline{3} 1 \frac{1}{3}} H^{\dagger}$ \\
\hline 2-iii-b & $(d \bar{e})(d)(\bar{u})(\bar{u} \bar{e})$ & 11 & $(\overline{3}, 2)_{-1 / 6}$ & $(\overline{6}, 2)_{+1 / 6}$ & $(\overline{3}, 1)_{+1 / 3}$ & $\mathrm{~T} \nu$-1-ii & $S_{\overline{3} 2-\frac{1}{6}}^{\dagger} S_{\overline{3} 1 \frac{1}{3}} H^{\dagger}$ \\
\hline 2-iii-b & $(d \bar{e})(d)(\bar{u})(\bar{u} \bar{e})$ & 11 & $(\overline{3}, 2)_{-1 / 6}$ & $(3,2)_{+1 / 6}$ & $(\overline{3}, 3)_{+1 / 3}$ & $\mathrm{~T} \nu$-1-ii & $S_{\overline{3} 2-\frac{1}{6}}^{\dagger} S_{\overline{3} 3 \frac{1}{3}} H^{\dagger}$ \\
\hline 2-iii-b & $(d \bar{e})(d)(\bar{u})(\bar{u} \bar{e})$ & 11 & $(\overline{3}, 2)_{-1 / 6}$ & $(\overline{6}, 2)_{+1 / 6}$ & $(\overline{3}, 3)_{+1 / 3}$ & $\mathrm{~T} \nu$-1-ii & $S_{\overline{3} 2-\frac{1}{6}}^{\dagger} S_{\overline{3} 3 \frac{1}{3}} H^{\dagger}$ \\
\hline 2 -iii-b & $(d \bar{e})(d)(\bar{u})(\bar{u} \bar{e})$ & 14 & $(\overline{3}, 2)_{-1 / 6}$ & $(3,1)_{-1 / 3}$ & $(\overline{3}, 1)_{+1 / 3}$ & $\mathrm{~T} \nu$-1-ii & $S_{\overline{3} 2-\frac{1}{6}}^{\dagger} S_{\overline{3} 1 \frac{1}{3}} H^{\dagger}$ \\
\hline 2-iii-b & $(d \bar{e})(d)(\bar{u})(\bar{u} \bar{e})$ & 14 & $(\overline{3}, 2)_{-1 / 6}$ & $(\overline{6}, 1)_{-1 / 3}$ & $(\overline{3}, 1)_{+1 / 3}$ & $\mathrm{~T} \nu$-1-ii & $S_{\overline{3} 2-\frac{1}{6}}^{\dagger} S_{\overline{3} 1 \frac{1}{3}} H^{\dagger}$ \\
\hline 2-iii-b & $(d \bar{e})(d)(\bar{u})(\bar{u} \bar{e})$ & 14 & $(\overline{3}, 2)_{-1 / 6}$ & $(3,3)_{-1 / 3}$ & $(\overline{3}, 3)_{+1 / 3}$ & $\mathrm{~T} \nu$-1-ii & $S_{\overline{3} 2-\frac{1}{6}}^{\dagger} S_{\overline{3} 3 \frac{1}{3}} H^{\dagger}$ \\
\hline 2 -iii-b & $(d \bar{e})(d)(\bar{u})(\bar{u} \bar{e})$ & 14 & $(\overline{3}, 2)_{-1 / 6}$ & $(\overline{6}, 3)_{-1 / 3}$ & $(\overline{3}, 3)_{+1 / 3}$ & $\mathrm{~T} \nu$-1-ii & $S_{\overline{3} 2-\frac{1}{6}}^{\dagger} S_{\overline{3} 3 \frac{1}{3}} H^{\dagger}$ \\
\hline $3-\mathrm{i}$ & $(\bar{u} \bar{u})(\bar{e})(\bar{e})(d d)$ & 11 & $(6,3)_{+1 / 3}$ & $(6,2)_{-1 / 6}$ & $(6,1)_{-2 / 3}$ & $\mathrm{~T} \nu-3$ & $S_{63 \frac{1}{3}} S_{61-\frac{2}{3}}^{\dagger} H^{\dagger} H^{\dagger}$ \\
\hline $3-\mathrm{i}$ & $(\bar{u} \bar{u})(\bar{e})(\bar{e})(d d)$ & 12 & $(6,1)_{+4 / 3}$ & $(6,2)_{+5 / 6}$ & $(6,3)_{+1 / 3}$ & $\mathrm{~T} \nu-3$ & $S_{61 \frac{4}{3}} S_{63 \frac{1}{3}}^{\dagger} H^{\dagger} H^{\dagger}$ \\
\hline 4 -ii-a & $(\bar{u} \bar{u})(d)(\bar{e})(d \bar{e})$ & 11 & $(6,3)_{+1 / 3}$ & $(3,3)_{+2 / 3}$ & $(3,2)+1 / 6$ & $\mathrm{~T} \nu$-1-iii & $\bar{Q} \psi_{33 \frac{2}{3}} H^{\dagger}$ \\
\hline 5 -ii-b & $(\bar{u} \bar{e})(\bar{e})(\bar{u})(d d)$ & 11 & $(3,1)_{-1 / 3}$ & $(3,2)_{-5 / 6}$ & $(6,1)_{-2 / 3}$ & $\mathrm{~T} \nu-1-\mathrm{iii}$ & $\bar{d}_{R}^{c} \psi_{32-\frac{5}{6}}^{c} H^{\dagger}$ \\
\hline 5 -ii-b & $(\bar{u} \bar{e})(\bar{e})(\bar{u})(d d)$ & 11 & $(3,3)_{-1 / 3}$ & $(3,2)_{-5 / 6}$ & $(6,1)_{-2 / 3}$ & $\mathrm{~T} \nu$-1-iii & $\bar{d}_{R}^{c} \psi_{32-\frac{5}{6}}^{c} H^{\dagger}$ \\
\hline
\end{tabular}

Table 2. Decompositions that generate neutrino masses at 1-loop. The naming convention of 1loop neutrino mass diagram, which is used in "Diagram" column, follows ref. [77] and is also shown in figure (4). For the decompositions in this table, unless some severe fine-tuning of parameters is done, the mass mechanism of double beta decay will dominate over the short-range contributions. 


\begin{tabular}{|c|c|c|c|c|c|c|c|}
\hline T-II \# & Op. & BL \# & $S$ & $S^{\prime}$ & $S^{\prime \prime}$ & Diagram & Add. Int. \\
\hline 2 & $(\bar{u} d)(\bar{u} \bar{e})(d \bar{e})$ & 11,14 & $(1,2)_{+1 / 2}$ & $(3,1)_{-1 / 3}$ & $(\overline{3}, 2)_{-1 / 6}$ & $\mathrm{~T} \nu$-1-ii & $S_{\overline{3} 2-\frac{1}{6}}^{\dagger} S_{31-\frac{1}{3}}^{\dagger} H^{\dagger}$ \\
\hline 2 & $(\bar{u} d)(\bar{u} \bar{e})(d \bar{e})$ & 11,14 & $(1,2)_{+1 / 2}$ & $(3,3)_{-1 / 3}$ & $(\overline{3}, 2)_{-1 / 6}$ & $\mathrm{~T} \nu$-1-ii & $S_{\overline{3} 2-\frac{1}{6}}^{\dagger} S_{33-\frac{1}{3}}^{\dagger} H^{\dagger}$ \\
\hline 2 & $(\bar{u} d)(\bar{u} \bar{e})(d \bar{e})$ & 11,14 & $(8,2)_{+1 / 2}$ & $(3,1)_{-1 / 3}$ & $(\overline{3}, 2)_{-1 / 6}$ & $\mathrm{~T} \nu$-1-ii & $S_{\overline{3} 2-\frac{1}{6}}^{\dagger} S_{31-\frac{1}{3}}^{\dagger} H^{\dagger}$ \\
\hline 2 & $(\bar{u} d)(\bar{u} \bar{e})(d \bar{e})$ & 11,14 & $(8,2)_{+1 / 2}$ & $(3,3)_{-1 / 3}$ & $(\overline{3}, 2)_{-1 / 6}$ & $\mathrm{~T} \nu$-1-ii & $S_{\overline{3} 2-\frac{1}{6}}^{\dagger} S_{33-\frac{1}{3}}^{\dagger} H^{\dagger}$ \\
\hline
\end{tabular}

Table 3. Decompositions (T-II) that generate neutrino mass at 1-loop, which are continued from table 2. For the decompositions in this table, unless some severe fine-tuning of parameters is done, the mass mechanism of double beta decay will dominate over the short-range contributions.

\begin{tabular}{|ccccccc|}
\hline T-I \# & Op. & BL \# & $S$ & $\psi$ & $S^{\prime}$ & Diagram \\
\hline 2-iii-a & $(d \bar{e})(\bar{u})(d)(\bar{u} \bar{e})$ & 19 & $(\overline{3}, 2)_{-1 / 6}$ & $(8,1)_{0}$ & $(\overline{3}, 1)_{+1 / 3}$ & PTBM-1 \\
\hline 4-i & $(d \bar{e})(\bar{u})(\bar{u})(d \bar{e})$ & 11 & $(\overline{3}, 2)_{-1 / 6}$ & $(8,1)_{0}$ & $(3,2)_{+1 / 6}$ & PTBM-1 \\
\hline 4-i & $(d \bar{e})(\bar{u})(\bar{u})(d \bar{e})$ & 11 & $(\overline{3}, 2)_{-1 / 6}$ & $(8,3)_{0}$ & $(3,2)_{+1 / 6}$ & PTBM-1 \\
\hline 4-ii-b & $(\bar{u} \bar{u})(\bar{e})(d)(d \bar{e})$ & 11 & $(6,3)_{+1 / 3}$ & $(6,2)_{-1 / 6}$ & $(3,2)_{+1 / 6}$ & PTBM-4 \\
\hline 5-i & $(\bar{u} \bar{e})(d)(d)(\bar{u} \bar{e})$ & 11,19 & $(3,1)_{-1 / 3}$ & $(8,1)_{0}$ & $(\overline{3}, 1)_{+1 / 3}$ & PTBM-1 \\
\hline 5-i & $(\bar{u} \bar{e})(d)(d)(\bar{u} \bar{e})$ & 11 & $(3,3)_{-1 / 3}$ & $(8,3)_{0}$ & $(\overline{3}, 3)_{+1 / 3}$ & PTBM-1 \\
\hline 5-ii-a & $(\bar{u} \bar{e})(\bar{u})(\bar{e})(d d)$ & 11 & $(3,1)_{-1 / 3}$ & $(6,2)_{-1 / 6}$ & $(6,1)_{-2 / 3}$ & PTBM-4 \\
\hline 5-ii-a & $(\bar{u} \bar{e})(\bar{u})(\bar{e})(d d)$ & 11 & $(3,3)_{-1 / 3}$ & $(6,2)_{-1 / 6}$ & $(6,1)_{-2 / 3}$ & PTBM-4 \\
\hline
\end{tabular}

\begin{tabular}{|ccccccc|}
\hline T-II \# & Op. & BL \# & $S$ & $S^{\prime}$ & $S^{\prime \prime}$ & Diagram \\
\hline 4 & $(\bar{u} \bar{u})(d \bar{e})(d \bar{e})$ & 11 & $(6,3)_{+1 / 3}$ & $(\overline{3}, 2)_{-1 / 6}$ & $(\overline{3}, 2)_{-1 / 6}$ & CLBZ-1 \\
\hline 5 & $(\bar{u} \bar{e})(\bar{u} \bar{e})(d d)$ & 11 & $(3,1)_{-1 / 3}$ & $(3,1)_{-1 / 3}$ & $(\overline{6}, 1)_{+2 / 3}$ & CLBZ-1 \\
\hline 5 & $(\bar{u} \bar{e})(\bar{u} \bar{e})(d d)$ & 11 & $(3,3)_{-1 / 3}$ & $(3,3)_{-1 / 3}$ & $(\overline{6}, 1)_{+2 / 3}$ & CLBZ-1 \\
\hline
\end{tabular}

Table 4. Decompositions that generate the $d=5$ neutrino mass operator $L L H H$ at 2-Loop. We follow the naming convention used in [62]. Although the effective operator of BL \#19 can generate neutrino mass only at the 3-loop level [35, 36], the decompositions of BL \#19 listed in this table generate not only the BL \#19 but also the "associated" BL \#11 operator and thus are classified as 2-loop neutrino mass models. For the decompositions in this table, the mass mechanism of double beta decay and the short-range contributions can be comparable. 


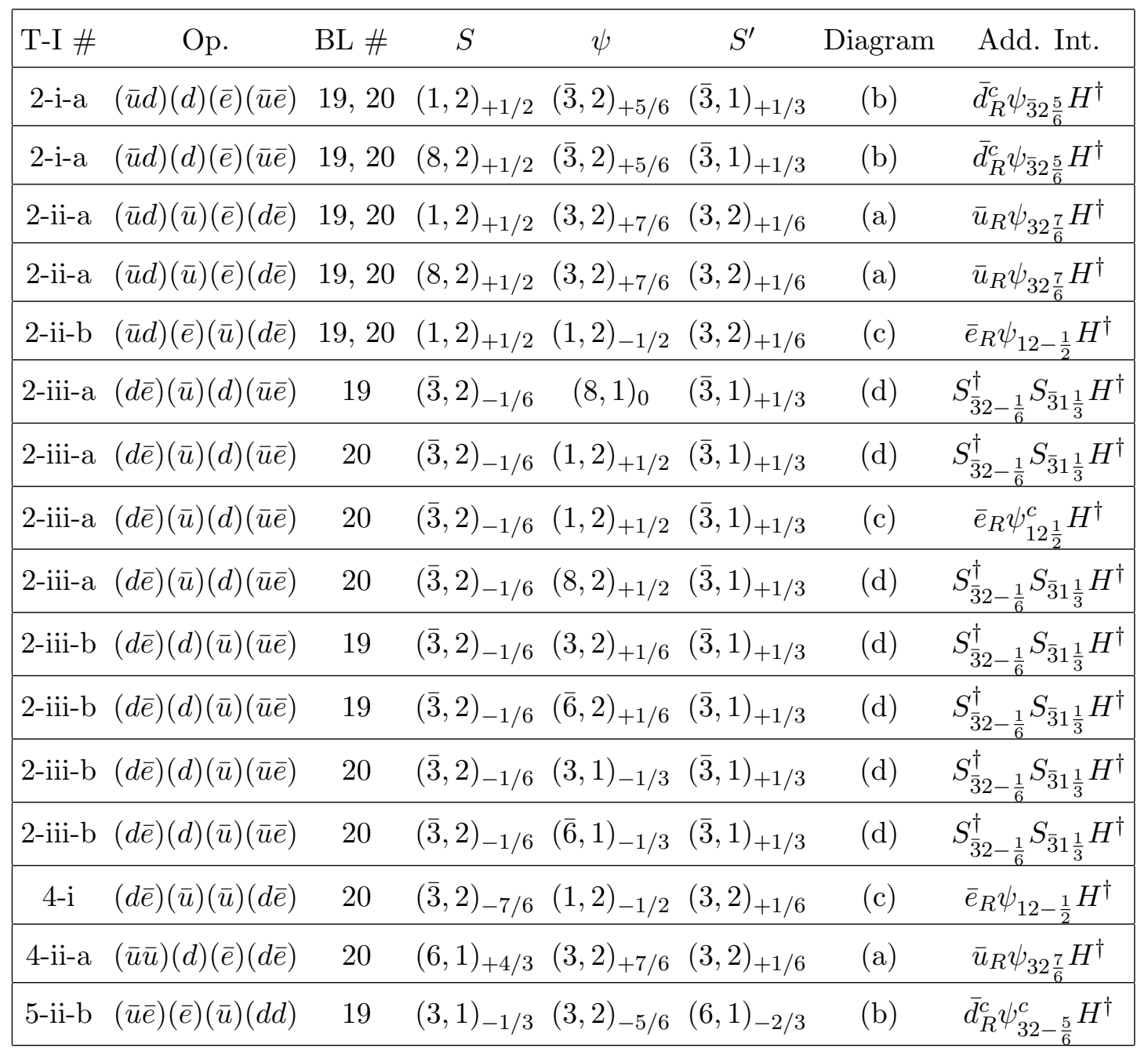

\begin{tabular}{|cccccccc|}
\hline T-II \# & Op. & BL \# & $S$ & $S^{\prime}$ & $S^{\prime \prime}$ & Diagram & Add. Int. \\
\hline 2 & $(\bar{u} d)(\bar{u} \bar{e})(d \bar{e})$ & 19,20 & $(1,2)_{+1 / 2}$ & $(3,1)_{-1 / 3}$ & $(\overline{3}, 2)_{-1 / 6}$ & $(\mathrm{~d})$ & $S_{\overline{3} 2-\frac{1}{6}}^{\dagger} S_{31-\frac{1}{3}}^{\dagger} H^{\dagger}$ \\
\hline 2 & $(\bar{u} d)(\bar{u} \bar{e})(d \bar{e})$ & 19,20 & $(8,2)_{+1 / 2}$ & $(3,1)_{-1 / 3}$ & $(\overline{3}, 2)_{-1 / 6}$ & $(\mathrm{~d})$ & $S_{\overline{3} 2-\frac{1}{6}}^{\dagger} S_{31-\frac{1}{3}}^{\dagger} H^{\dagger}$ \\
\hline
\end{tabular}

Table 5. Decompositions that generate $d=7$ neutrino mass operator $L L H H H H^{\dagger}$ at the 2 loop level. The topologies of the neutrino mass diagrams in the column of "Diagram" are shown in figure 18. For the decompositions in this table, unless some severe fine-tuning of parameters is done, the short-range contributions will dominate over the mass mechanism of double beta decay. 


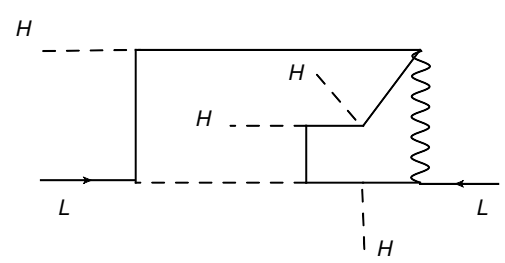

(a)

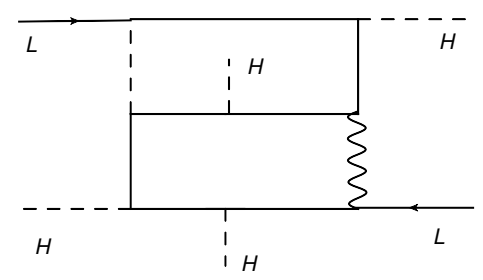

(c)

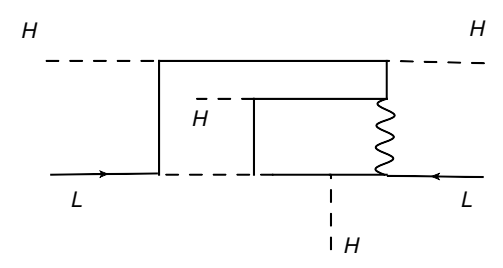

(b)

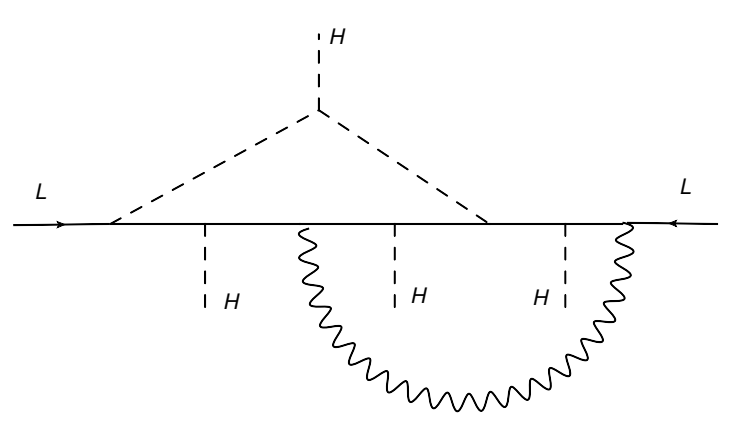

(d)

Figure 18. Dimension $7(d=7)$ neutrino mass diagrams generated by the decompositions listed in table 5 .

\begin{tabular}{|cccccc|}
\hline T-I \# & Op. & BL \# & $S$ & $\psi$ & $S^{\prime}$ \\
\hline 2-ii-b & $(\bar{u} d)(\bar{e})(\bar{u})(d \bar{e})$ & 19,20 & $(8,2)_{+1 / 2}$ & $(8,2)_{-1 / 2}$ & $(3,2)_{+1 / 6}$ \\
\hline 4-i & $(d \bar{e})(\bar{u})(\bar{u})(d \bar{e})$ & 20 & $(\overline{3}, 2)_{-7 / 6}$ & $(8,2)_{-1 / 2}$ & $(3,2)_{+1 / 6}$ \\
\hline 4-ii-a & $(\bar{u} \bar{u})(d)(\bar{e})(d \bar{e})$ & 20 & $(6,1)_{+4 / 3}$ & $(3,1)_{+5 / 3}$ & $(3,2)_{+7 / 6}$ \\
\hline 4-ii-b & $(\bar{u} \bar{u})(\bar{e})(d)(d \bar{e})$ & 20 & $(6,1)_{+4 / 3}$ & $(6,1)_{+1 / 3}$ & $(3,2)_{+1 / 6}$ \\
\hline 4-ii-b & $(\bar{u} \bar{u})(\bar{e})(d)(d \bar{e})$ & 20 & $(6,1)_{+4 / 3}$ & $(6,2)_{+5 / 6}$ & $(3,2)_{+7 / 6}$ \\
\hline 5-ii-a & $(\bar{u} \bar{e})(\bar{u})(\bar{e})(d d)$ & 19 & $(3,1)_{-1 / 3}$ & $(6,1)_{+1 / 3}$ & $(6,1)_{-2 / 3}$ \\
\hline 5-ii-a & $(\bar{u} \bar{e})(\bar{u})(\bar{e})(d d)$ & 19 & $(3,1)_{-1 / 3}$ & $(6,2)_{-1 / 6}$ & $(6,1)_{-2 / 3}$ \\
\hline 5-ii-b & $(\bar{u} \bar{e})(\bar{e})(\bar{u})(d d)$ & 19 & $(3,1)_{-1 / 3}$ & $(3,1)_{-4 / 3}$ & $(6,1)_{-2 / 3}$ \\
\hline
\end{tabular}

\begin{tabular}{|cccccc|}
\hline T-II \# & Op. & BL \# & $S$ & $S^{\prime}$ & $S^{\prime \prime}$ \\
\hline 4 & $(\bar{u} \bar{u})(d \bar{e})(d \bar{e})$ & 20 & $(6,1)_{+4 / 3}$ & $(\overline{3}, 2)_{-7 / 6}$ & $(\overline{3}, 2)_{-1 / 6}$ \\
\hline 5 & $(\bar{u} \bar{e})(\bar{u} \bar{e})(d d)$ & 19 & $(3,1)_{-1 / 3}$ & $(3,1)_{-1 / 3}$ & $(\overline{6}, 1)_{+2 / 3}$ \\
\hline
\end{tabular}

Table 6. Decompositions that generate neutrino masses at 3-loop. Some example diagrams are given in the main text. For the decompositions in this table, unless some severe fine-tuning of parameters is done, the short-range contributions will dominate over the mass mechanism of double beta decay. 


\begin{tabular}{|cccccc|}
\hline T-I \# & Op. & BL $\#$ & $S$ & $\psi$ & $S^{\prime}$ \\
\hline 3-i & $(\bar{u} \bar{u})(\bar{e})(\bar{e})(d d)$ & - & $(6,1)_{+4 / 3}$ & $(6,1)_{+1 / 3}$ & $(6,1)_{-2 / 3}$ \\
\hline 3-ii & $(\bar{u} \bar{u})(d)(d)(\bar{e} \bar{e})$ & - & $(6,1)_{+4 / 3}$ & $(3,1)_{+5 / 3}$ & $(1,1)_{+2}$ \\
\hline 3-iii & $(d d)(\bar{u})(\bar{u})(\bar{e} \bar{e})$ & - & $(\overline{6}, 1)_{+2 / 3}$ & $(\overline{3}, 1)_{+4 / 3}$ & $(1,1)_{+2}$ \\
\hline 5-i & $(\bar{u} \bar{e})(d)(d)(\bar{u} \bar{e})$ & - & $(3,1)_{-1 / 3}$ & $(8,1)_{0}$ & $(\overline{3}, 1)_{+1 / 3}$ \\
\hline 5-ii-a & $(\bar{u} \bar{e})(\bar{u})(\bar{e})(d d)$ & - & $(3,1)_{-1 / 3}$ & $(6,1)_{+1 / 3}$ & $(6,1)_{-2 / 3}$ \\
\hline 5-ii-b & $(\bar{u} \bar{e})(\bar{e})(\bar{u})(d d)$ & - & $(3,1)_{-1 / 3}$ & $(3,1)_{-4 / 3}$ & $(6,1)_{-2 / 3}$ \\
\hline
\end{tabular}

\begin{tabular}{|cccccc|}
\hline T-II \# & Op. & BL $\#$ & $S$ & $S^{\prime}$ & $S^{\prime \prime}$ \\
\hline 3 & $(\bar{u} \bar{u})(d d)(\bar{e} \bar{e})$ & - & $(6,1)_{+4 / 3}$ & $(\overline{6}, 1)_{+2 / 3}$ & $(1,1)_{-2}$ \\
\hline 5 & $(\bar{u} \bar{e})(\bar{u} \bar{e})(d d)$ & - & $(3,1)_{-1 / 3}$ & $(3,1)_{-1 / 3}$ & $(\overline{6}, 1)_{+2 / 3}$ \\
\hline
\end{tabular}

Table 7. Decompositions that generate neutrino masses at 4-loop. For the decompositions in this table, the short-range contributions will dominate over the mass mechanism of double beta decay.

Open Access. This article is distributed under the terms of the Creative Commons Attribution License (CC-BY 4.0), which permits any use, distribution and reproduction in any medium, provided the original author(s) and source are credited.

\section{References}

[1] F.F. Deppisch, M. Hirsch and H. Päs, Neutrinoless Double Beta Decay and Physics Beyond the Standard Model, J. Phys. G 39 (2012) 124007 [arXiv:1208.0727] [InSPIRE].

[2] GERDA collaboration, M. Agostini et al., Results on Neutrinoless Double- $\beta$ Decay of ${ }^{76}$ Ge from Phase I of the GERDA Experiment, Phys. Rev. Lett. 111 (2013) 122503 [arXiv: 1307.4720] [INSPIRE].

[3] EXO-200 collaboration, J.B. Albert et al., Search for Majorana neutrinos with the first two years of EXO-200 data, Nature 510 (2014) 229 [arXiv:1402.6956] [INSPIRE].

[4] KamLAND-Zen collaboration, I. Shimizu, Results from KamLAND-Zen, presented at Neutrino 2014, Boston, U.S.A., June 2-7 2014.

[5] KamLAND-Zen collaboration, A. Gando et al., Limit on Neutrinoless $\beta \beta$ Decay of ${ }^{136}$ Xe from the First Phase of KamLAND-Zen and Comparison with the Positive Claim in ${ }^{76} \mathrm{Ge}$, Phys. Rev. Lett. 110 (2013) 062502 [arXiv:1211.3863] [INSPIRE].

[6] K. Muto, E. Bender and H.V. Klapdor, Nuclear Structure Effects on the Neutrinoless Double Beta Decay, Z. Phys. A 334 (1989) 187 [InSPIRE].

[7] A. Faessler, V. Rodin and F. Šimkovic, Nuclear matrix elements for neutrinoless double-beta decay and double-electron capture, J. Phys. G 39 (2012) 124006 [arXiv:1206.0464] [INSPIRE].

[8] J. Menendez, A. Poves, E. Caurier and F. Nowacki, Neutrinoless double beta decay: The nuclear matrix elements revisited, J. Phys. Conf. Ser. 312 (2011) 072005 [INSPIRE]. 
[9] R.N. Mohapatra and G. Senjanović, Neutrino masses and mixings in gauge models with spontaneous parity violation, Phys. Rev. D 23 (1981) 165 [INSPIRE].

[10] M. Doi, T. Kotani and E. Takasugi, Double beta decay and Majorana neutrino, Prog. Theor. Phys. Suppl. 83 (1985) 1 [INSPIRE].

[11] R.N. Mohapatra, New contributions to neutrinoless double beta decay in supersymmetric theories, Phys. Rev. D 34 (1986) 3457 [InSPIRE].

[12] M. Hirsch, H.V. Klapdor-Kleingrothaus and S.G. Kovalenko, New constraints on R-parity broken supersymmetry from neutrinoless double beta decay, Phys. Rev. Lett. 75 (1995) 17 [INSPIRE].

[13] A. Faessler, S.G. Kovalenko and F. Šimkovic, Bilinear R-parity violation in neutrinoless double beta decay, Phys. Rev. D 58 (1998) 055004 [hep-ph/9712535] [INSPIRE].

[14] M. Hirsch and J.W.F. Valle, Neutrinoless double beta decay in supersymmetry with bilinear $R$ parity breaking, Nucl. Phys. B 557 (1999) 60 [hep-ph/9812463] [INSPIRE].

[15] M. Hirsch, H.V. Klapdor-Kleingrothaus and S.G. Kovalenko, New leptoquark mechanism of neutrinoless double beta decay, Phys. Rev. D 54 (1996) 4207 [hep-ph/9603213] [INSPIRE].

[16] S. Choubey, M. Duerr, M. Mitra and W. Rodejohann, Lepton number and lepton flavor violation through color octet states, JHEP 05 (2012) 017 [arXiv:1201.3031] [INSPIRE].

[17] B. Brahmachari and E. Ma, Neutrinoless double beta decay with negligible neutrino mass, Phys. Lett. B 536 (2002) 259 [hep-ph/0202262] [INSPIRE].

[18] P.-H. Gu, Significant neutrinoless double beta decay with quasi-Dirac neutrinos, Phys. Rev. D 85 (2012) 093016 [arXiv:1101.5106] [INSPIRE].

[19] M. Kohda, H. Sugiyama and K. Tsumura, Lepton number violation at the LHC with leptoquark and diquark, Phys. Lett. B 718 (2013) 1436 [arXiv:1210.5622] [INSPIRE].

[20] N. Steinbrink et al., Neutrino mass sensitivity by MAC-E-Filter based time-of-flight spectroscopy with the example of KATRIN, New J. Phys. 15 (2013) 113020 [arXiv: 1308.0532] [INSPIRE].

[21] J. Lesgourgues and S. Pastor, Massive neutrinos and cosmology, Phys. Rept. 429 (2006) 307 [astro-ph/0603494] [INSPIRE].

[22] S. Hannestad, Neutrino physics from precision cosmology, Prog. Part. Nucl. Phys. 65 (2010) 185 [arXiv: 1007.0658] [INSPIRE].

[23] Y.Y.Y. Wong, Neutrino mass in cosmology: status and prospects, Ann. Rev. Nucl. Part. Sci. 61 (2011) 69 [arXiv:1111.1436] [INSPIRE].

[24] A. Ali, A.V. Borisov and D.V. Zhuridov, Probing new physics in the neutrinoless double beta decay using electron angular correlation, Phys. Rev. D 76 (2007) 093009 [arXiv:0706.4165] [INSPIRE].

[25] SuperNEMO collaboration, R. Arnold et al., Probing new physics models of neutrinoless double beta decay with SuperNEMO, Eur. Phys. J. C 70 (2010) 927 [arXiv:1005.1241] [INSPIRE].

[26] M. Hirsch, K. Muto, T. Oda and H.V. Klapdor-Kleingrothaus, Nuclear structure calculations of $\beta^{+} \beta^{+}, \beta^{+} / E C$ and EC/EC decay matrix elements, Z. Phys. A 347 (1994) 151 [INSPIRE].

[27] H. Päs, M. Hirsch, H.V. Klapdor-Kleingrothaus and S.G. Kovalenko, Towards a superformula for neutrinoless double beta decay, Phys. Lett. B 453 (1999) 194 [INSPIRE]. 
[28] H. Päs, M. Hirsch, H.V. Klapdor-Kleingrothaus and S.G. Kovalenko, A superformula for neutrinoless double beta decay. 2. The short range part, Phys. Lett. B 498 (2001) 35 [hep-ph/0008182] [INSPIRE].

[29] J.C. Helo, M. Hirsch, S.G. Kovalenko and H. Päs, Neutrinoless double beta decay and lepton number violation at the LHC, Phys. Rev. D 88 (2013) 011901 [arXiv:1303.0899] [INSPIRE].

[30] J.C. Helo, M. Hirsch, H. Päs and S.G. Kovalenko, Short-range mechanisms of neutrinoless double beta decay at the LHC, Phys. Rev. D 88 (2013) 073011 [arXiv:1307.4849] [InSPIRE].

[31] F. Bonnet, M. Hirsch, T. Ota and W. Winter, Systematic decomposition of the neutrinoless double beta decay operator, JHEP 03 (2013) 055 [Erratum ibid. 04 (2014) 090] [arXiv: 1212.3045] [INSPIRE].

[32] J. Schechter and J.W.F. Valle, Neutrinoless double beta decay in $\mathrm{SU}(2) \times \mathrm{U}(1)$ theories, Phys. Rev. D 25 (1982) 2951 [inSPIRE].

[33] M. Hirsch, S.G. Kovalenko and I. Schmidt, Extended black box theorem for lepton number and flavor violating processes, Phys. Lett. B 642 (2006) 106 [hep-ph/0608207] [INSPIRE].

[34] M. Duerr, M. Lindner and A. Merle, On the quantitative impact of the Schechter-Valle theorem, JHEP 06 (2011) 091 [arXiv:1105.0901] [INSPIRE].

[35] K.S. Babu and C.N. Leung, Classification of effective neutrino mass operators, Nucl. Phys. B 619 (2001) 667 [hep-ph/0106054] [INSPIRE].

[36] A. de Gouvêa and J. Jenkins, A Survey of Lepton Number Violation Via Effective Operators, Phys. Rev. D 77 (2008) 013008 [arXiv:0708.1344] [INSPIRE].

[37] P.W. Angel, N.L. Rodd and R.R. Volkas, Origin of neutrino masses at the LHC: $\Delta L=2$ effective operators and their ultraviolet completions, Phys. Rev. D 87 (2013) 073007 [arXiv: 1212.6111] [INSPIRE].

[38] S. Weinberg, Baryon and Lepton Nonconserving Processes, Phys. Rev. Lett. 43 (1979) 1566 [INSPIRE].

[39] F. del Aguila, A. Aparici, S. Bhattacharya, A. Santamaria and J. Wudka, Effective Lagrangian approach to neutrinoless double beta decay and neutrino masses, JHEP 06 (2012) 146 [arXiv:1204.5986] [INSPIRE].

[40] D. Aristizabal Sierra, M. Hirsch and S.G. Kovalenko, Leptoquarks: neutrino masses and accelerator phenomenology, Phys. Rev. D 77 (2008) 055011 [arXiv:0710.5699] [INSPIRE].

[41] K.S. Babu and J. Julio, Radiative Neutrino Mass Generation through Vector-like Quarks, Phys. Rev. D 85 (2012) 073005 [arXiv:1112.5452] [inSPIRE].

[42] M. Blennow, E. Fernandez-Martinez, J. Lopez-Pavon and J. Menendez, Neutrinoless double beta decay in seesaw models, JHEP 07 (2010) 096 [arXiv: 1005.3240] [INSPIRE].

[43] M. Mitra, G. Senjanović and F. Vissani, Neutrinoless Double Beta Decay and Heavy Sterile Neutrinos, Nucl. Phys. B 856 (2012) 26 [arXiv:1108.0004] [INSPIRE].

[44] J. Lopez-Pavon, S. Pascoli and C.-f. Wong, Can heavy neutrinos dominate neutrinoless double beta decay?, Phys. Rev. D 87 (2013) 093007 [arXiv:1209.5342] [INSPIRE].

[45] J. Chakrabortty, H.Z. Devi, S. Goswami and S. Patra, Neutrinoless double- $\beta$ decay in TeV scale Left-Right symmetric models, JHEP 08 (2012) 008 [arXiv:1204.2527] [INSPIRE].

[46] W.-C. Huang and J. Lopez-Pavon, On neutrinoless double beta decay in the minimal left-right symmetric model, Eur. Phys. J. C 74 (2014) 2853 [arXiv:1310.0265] [INSPIRE]. 
[47] P.S. Bhupal Dev, S. Goswami, M. Mitra and W. Rodejohann, Constraining Neutrino Mass from Neutrinoless Double Beta Decay, Phys. Rev. D 88 (2013) 091301 [arXiv:1305.0056] [INSPIRE].

[48] S. Pascoli, M. Mitra and S. Wong, Effect of cancellation in neutrinoless double beta decay, Phys. Rev. D 90 (2014) 093005 [arXiv: 1310.6218] [InSPIRE].

[49] A. Merle and V. Niro, Influence of a keV sterile neutrino on neutrinoless double beta decay: How things changed in recent years, Phys. Rev. D 88 (2013) 113004 [arXiv:1302.2032] [INSPIRE].

[50] P.S.B. Dev, S. Goswami and M. Mitra, TeV Scale Left-Right Symmetry and Large Mixing Effects in Neutrinoless Double Beta Decay, arXiv:1405.1399 [INSPIRE].

[51] Y.F. Li and S.-s. Liu, Vanishing effective mass of the neutrinoless double beta decay including light sterile neutrinos, Phys. Lett. B 706 (2012) 406 [arXiv:1110.5795] [InSPIRE].

[52] I. Girardi, A. Meroni and S.T. Petcov, Neutrinoless Double Beta Decay in the Presence of Light Sterile Neutrinos, JHEP 11 (2013) 146 [arXiv:1308.5802] [INSPIRE].

[53] A. Meroni and E. Peinado, The quest for neutrinoless double beta decay: Pseudo-Dirac, Majorana and sterile neutrinos, Phys. Rev. D 90 (2014) 053002 [arXiv:1406.3990] [INSPIRE].

[54] A. Faessler, M. González, S.G. Kovalenko and F. Šimkovic, Arbitrary mass Majorana neutrinos in neutrinoless double beta decay, Phys. Rev. D 90 (2014) 096010 [arXiv: 1408.6077] [INSPIRE].

[55] B. Dudley and C. Kolda, The Effect of Quark Sector Minimal Flavor Violation on Neutrinoless Double Beta Decay, Phys. Rev. D 79 (2009) 013014 [arXiv:0810.2997] [INSPIRE].

[56] K.S. Babu and R.N. Mohapatra, New vector-scalar contributions to neutrinoless double beta decay and constraints on R-parity violation, Phys. Rev. Lett. 75 (1995) 2276 [hep-ph/9506354] [INSPIRE].

[57] M. Hirsch, H.V. Klapdor-Kleingrothaus and S.G. Kovalenko, On the SUSY accompanied neutrino exchange mechanism of neutrinoless double beta decay, Phys. Lett. B 372 (1996) 181 [hep-ph/9512237] [INSPIRE].

[58] C.-S. Chen, C.Q. Geng and J.N. Ng, Unconventional Neutrino Mass Generation, Neutrinoless Double Beta Decays and Collider Phenomenology, Phys. Rev. D 75 (2007) 053004 [hep-ph/0610118] [INSPIRE].

[59] S.F. King, A. Merle and L. Panizzi, Effective theory of a doubly charged singlet scalar: complementarity of neutrino physics and the LHC, JHEP 11 (2014) 124 [arXiv:1406.4137] [INSPIRE].

[60] M. Gustafsson, J.M. No and M.A. Rivera, Predictive model for radiatively induced neutrino masses and mixings with dark matter, Phys. Rev. Lett. 110 (2013) 211802 [Erratum ibid. 112 (2014) 259902] [arXiv:1212.4806] [INSPIRE].

[61] M. Gustafsson, J.M. No and M.A. Rivera, Radiative neutrino mass generation linked to neutrino mixing and $0 \nu \beta \beta$-decay predictions, Phys. Rev. D 90 (2014) 013012 [arXiv: 1402.0515] [INSPIRE].

[62] D. Aristizabal Sierra, A. Degee, L. Dorame and M. Hirsch, Systematic classification of two-loop realizations of the Weinberg operator, JHEP 03 (2015) 040 [arXiv:1411.7038] [INSPIRE]. 
[63] E. Ma, Naturally small seesaw neutrino mass with no new physics beyond the TeV scale, Phys. Rev. Lett. 86 (2001) 2502 [hep-ph/0011121] [INSPIRE].

[64] E. Ma and M. Raidal, Neutrino mass, muon anomalous magnetic moment and lepton flavor nonconservation, Phys. Rev. Lett. 87 (2001) 011802 [hep-ph/0102255] [INSPIRE].

[65] N. Haba and M. Hirotsu, TeV-scale seesaw from a multi-Higgs model, Eur. Phys. J. C 69 (2010) 481 [arXiv: 1005.1372] [INSPIRE].

[66] N. Haba and T. Horita, Vacuum stability in neutrinophilic Higgs doublet model, Phys. Lett. B 705 (2011) 98 [arXiv:1107.3203] [INSPIRE].

[67] P. Minkowski, $\mu \rightarrow e \gamma$ at a rate of one out of $10^{9}$ muon decays?, Phys. Lett. B 67 (1977) 421 [INSPIRE].

[68] T. Yanagida, Horizontal symmetry and masses of neutrinos, Conf. Proc. C7902131 (1979) 95 [INSPIRE].

[69] M. Gell-Mann, P. Ramond, and R. Slansky, Complex spinors and unified theories, Conf. Proc. C790927 (1979) 315, Supergravity, P. van Nieuwenhuizen and D.Z. Freedman eds., North Holland Publishing Company (1979) [arXiv: 1306.4669] [INSPIRE].

[70] R.N. Mohapatra and G. Senjanović, Neutrino Mass and Spontaneous Parity Violation, Phys. Rev. Lett. 44 (1980) 912 [INSPIRE].

[71] M. Magg and C. Wetterich, Neutrino Mass Problem and Gauge Hierarchy, Phys. Lett. B 94 (1980) 61 [INSPIRE].

[72] J. Schechter and J.W.F. Valle, Neutrino masses in $\mathrm{SU}(2) \times \mathrm{U}(1)$ theories, Phys. Rev. D 22 (1980) 2227 [INSPIRE].

[73] C. Wetterich, Neutrino Masses and the Scale of B-L Violation, Nucl. Phys. B 187 (1981) 343 [INSPIRE].

[74] G. Lazarides, Q. Shafi and C. Wetterich, Proton lifetime and fermion masses in an $\mathrm{SO}(10)$ model, Nucl. Phys. B 181 (1981) 287 [InSPIRE].

[75] T.P. Cheng and L.-F. Li, Neutrino masses, mixings and oscillations in $\mathrm{SU}(2) \times \mathrm{U}(1)$ models of electroweak interactions, Phys. Rev. D 22 (1980) 2860 [InSPIRE].

[76] R. Foot, H. Lew, X.G. He and G.C. Joshi, Seesaw neutrino masses induced by a triplet of leptons, Z. Phys. C 44 (1989) 441 [inSPIRE].

[77] F. Bonnet, M. Hirsch, T. Ota and W. Winter, Systematic study of the D $=5$ Weinberg operator at one-loop order, JHEP 07 (2012) 153 [arXiv: 1204.5862] [INSPIRE].

[78] E. Ma, Pathways to naturally small neutrino masses, Phys. Rev. Lett. 81 (1998) 1171 [hep-ph/9805219] [INSPIRE].

[79] M. Hirsch, H.V. Klapdor-Kleingrothaus and S.G. Kovalenko, New low-energy leptoquark interactions, Phys. Lett. B 378 (1996) 17 [hep-ph/9602305] [INSPIRE].

[80] U. Mahanta, Neutrino masses and mixing angles from leptoquark interactions, Phys. Rev. D 62 (2000) 073009 [hep-ph/9909518] [INSPIRE].

[81] Y. Farzan, S. Pascoli and M.A. Schmidt, Recipes and Ingredients for Neutrino Mass at Loop Level, JHEP 03 (2013) 107 [arXiv:1208.2732] [INSPIRE].

[82] P.W. Angel, Y. Cai, N.L. Rodd, M.A. Schmidt and R.R. Volkas, Testable two-loop radiative neutrino mass model based on an $L L Q d^{c} Q d^{c}$ effective operator, JHEP 10 (2013) 118 [Erratum ibid. 11 (2014) 092] [arXiv:1308.0463] [INSPIRE]. 
[83] D.V. Forero, M. Tortola and J.W.F. Valle, Neutrino oscillations refitted, Phys. Rev. D 90 (2014) 093006 [arXiv: 1405.7540] [INSPIRE].

[84] L. Lavoura, General formulae for $f(1) \rightarrow f(2)$ gamma, Eur. Phys. J. C 29 (2003) 191 [hep-ph/0302221] [INSPIRE].

[85] KamLAnD-Zen collaboration, A. Gando et al., Measurement of the double- $\beta$ decay half-life of ${ }^{136}$ Xe with the KamLAND-Zen experiment, Phys. Rev. C 85 (2012) 045504 [arXiv: 1201.4664] [INSPIRE].

[86] CMS collaboration, Search for pair-production of first generation scalar leptoquarks in pp collisions at $\sqrt{s}=8 \mathrm{TeV}$, CMS-PAS-EXO-12-041.

[87] CMS Collaboration, Search for pair-production of second generation leptoquarks in $8 \mathrm{TeV}$ proton-proton collisions, CMS-PAS-EXO-12-042.

[88] CMS collaboration, Search for pair-production of third-generation scalar leptoquarks and top squarks in proton-proton collisions at $\sqrt{s}=8 \mathrm{TeV}$, Phys. Lett. B 739 (2014) 229 [arXiv: 1408.0806] [INSPIRE].

[89] MEG collaboration, J. Adam et al., New constraint on the existence of the $\mu^{+} \rightarrow e^{+} \gamma$ decay, Phys. Rev. Lett. 110 (2013) 201801 [arXiv:1303.0754] [INSPIRE].

[90] J.A. Casas and A. Ibarra, Oscillating neutrinos and $\mu \rightarrow e, \gamma$, Nucl. Phys. B 618 (2001) 171 [hep-ph/0103065] [INSPIRE]. 